\title{
SOME GENERALIZATIONS OF THE SECOND MAIN THEOREM INTERSECTING HYPERSURFACES*
}

\author{
MIN RUt
}

Dedicated to Professor Stephen Yau on the occasion of his 60th birthday

\begin{abstract}
In [18], the author established a defect relation for holomorphic mappings from $\mathbb{C}$ into the projective variety $X \subset \mathbb{P}^{N}(\mathbb{C})$ intersecting divisors of $X$ coming from hypersurfaces in $\mathbb{P}^{N}$. The purpose of this paper is to do some further generalizations. It contains two major parts: (I) We extend the defect relation to effective divisors on $X$ with $\operatorname{Pic}(X)=\mathbb{Z}$. (II) We extend the defect relation to meromorphic mappings from parabolic manifolds into $X \subset \mathbb{P}^{N}$.
\end{abstract}

Key words. Second Main Theorem, Nevanlinna theory, meromorphic mappings, parabolic manifolds.

AMS subject classifications. $32 \mathrm{H} 30$.

1. Introduction. Let $f: \mathbb{C} \rightarrow \mathbb{P}^{n}(\mathbb{C})$ be a linearly non-degenerate holomorphic map, and $H_{j}, 1 \leq j \leq q$, be hyperplanes in $\mathbb{P}^{n}(\mathbb{C})$ in general position. In 1933, H. Cartan (see [4]) proved the defect relation (or a Second Main Theorem) $\sum_{j=1}^{q} \delta_{f}\left(H_{j}\right) \leq n+1$. Recently, the author (see [18]) extended H. Cartan's result to algebraically nondegenerate holomorphic curves $f: \mathbb{C} \rightarrow X \subset \mathbb{P}^{N}(\mathbb{C})$ and obtained $\sum_{j=1}^{q} \delta_{f}\left(D_{j}\right) \leq \operatorname{dim} X+1$, where $X$ is a smooth projective variety and $D_{1}, \ldots, D_{q}$ are hypersurfaces in $\mathbb{P}^{N}(\mathbb{C})$ located in general position in $X$.

To get a more precise statement, we introduce some standard notations in Nevanlinna theory: Let $f: \mathbb{C} \rightarrow \mathbb{P}^{N}(\mathbb{C})$ be a holomorphic map. Let $\mathbf{f}=\left(f_{0}, \ldots, f_{N}\right)$ be a reduced representative of $f$, where $f_{0}, \ldots, f_{N}$ are entire functions on $\mathbb{C}$ and have no common zeros. The Nevanlinna-Cartan characteristic function $T_{f}(r)$ is defined by

$$
T_{f}(r)=\frac{1}{2 \pi} \int_{0}^{2 \pi} \log \left\|\mathbf{f}\left(r e^{i \theta}\right)\right\| d \theta
$$

where

$$
\|\mathbf{f}(z)\|=\max \left\{\left|f_{0}(z)\right|, \ldots,\left|f_{N}(z)\right|\right\} .
$$

The above definition is independent, up to an additive constant, of the choice of the reduced representation of $f$. Let $D$ be a hypersurface in $\mathbb{P}^{N}(\mathbb{C})$ of degree $d$ and let $Q$ be the homogeneous polynomial of degree $d$ defining $D$. The proximity function is defined as

$$
m_{f}(r, D)=\int_{0}^{2 \pi} \log \frac{\left\|\mathbf{f}\left(r e^{i \theta}\right)\right\|^{d}\|Q\|}{\left|Q(\mathbf{f})\left(r e^{i \theta}\right)\right|} \frac{d \theta}{2 \pi}
$$

\footnotetext{
*Received February 7, 2013; accepted for publication August 15, 2013.

$\dagger$ Department of Mathematics, University of Houston, Houston, TX 77204, USA (minru@math. uh.edu). The author is supported in part by NSA grant H98230-11-1-0201.
} 
where $\|Q\|$ is the maximum of the absolute values of the coefficients of $Q$. To define the counting function, let $n_{f}(r, D)$ be the number of zeros of $Q \circ \mathbf{f}$ in the disk $|z|<r$, counting multiplicity. The counting function is then defined by

$$
N_{f}(r, D)=\int_{1}^{r} \frac{n_{f}(t, D)}{t} d t
$$

The Poisson-Jensen formula implies:

The First Main Theorem. Let $f: \mathbb{C} \rightarrow \mathbb{P}^{N}(\mathbb{C})$ be a holomorphic map, and let $D$ be a hypersurface in $\mathbb{P}^{N}(\mathbb{C})$ of degree $d$. If $f(\mathbb{C}) \not \subset D$, then for every real number $r$ with $0<r<\infty$,

$$
m_{f}(r, D)+N_{f}(r, D)=d T_{f}(r)+O(1)
$$

where $O(1)$ is a constant independent of $r$.

Let $X \subset \mathbb{P}^{N}(\mathbb{C})$ be a smooth complex projective variety of dimension $n \geq 1$. Let $D_{1}, \ldots, D_{q}$ be hypersurfaces in $\mathbb{P}^{N}(\mathbb{C})$, where $q>n$. The hypersurfaces $D_{1}, \ldots, D_{q}$ are said to be in general position in $X$ if for every subset $\left\{i_{0}, \ldots, i_{n}\right\} \subset\{1, \ldots, q\}$,

$$
X \cap \operatorname{supp} D_{i_{0}} \cap \cdots \cap \operatorname{supp} D_{i_{n}}=\emptyset,
$$

where $\operatorname{supp}(D)$ means the support of the divisor $D$. A map $f: \mathbb{C} \rightarrow X$ is said to be algebraically non-degenerate if the image of $f$ is not contained in any proper subvarieties of $X$. The following result, which extends the result of H. Cartan, was proved in [18].

TheOrem A [18]. Let $X \subset \mathbb{P}^{N}(\mathbb{C})$ be a smooth complex projective variety. Let $D_{1}, \ldots, D_{q}$ be hypersurfaces in $\mathbb{P}^{N}(\mathbb{C})$ of degree $d_{j}$, located in general position in $X$. Let $f: \mathbb{C} \rightarrow X$ be an algebraically non-degenerate holomorphic map. Then, for every $\epsilon>0$,

$$
\sum_{j=1}^{q} d_{j}^{-1} m_{f}\left(r, D_{j}\right) \leq(\operatorname{dim} X+1+\epsilon) T_{f}(r) \|_{E}
$$

where $\|_{E}$ means that the inequality holds for all $r$ except a set $E \subset(0,+\infty)$ with finite Lebesgue measure.

Define the defect of $f$ with respect to a hypersurface $D$ of degree $d$ by

$$
\delta_{f}(D)=\liminf _{r \rightarrow+\infty} \frac{m_{f}(r, D)}{d T_{f}(r)}=1-\limsup _{r \rightarrow+\infty} \frac{N_{f}(r, D)}{d T_{f}(r)} .
$$

Then Theorem A gives

Corollary A (Defect Relation). Under the same assumptions in Theorem A, we have

$$
\sum_{j=1}^{q} \delta_{f}\left(D_{j}\right) \leq \operatorname{dim} X+1
$$

The purpose of this paper is to generalize the above results. This paper contains two major parts: (I) We extend Theorem A to effective divisors on $X$ with $\operatorname{Pic}(X)=\mathbb{Z}$, where $\operatorname{Pic}(X)$ is the Picard group of $X$, by verifying that in the case $\operatorname{Pic}(X)=\mathbb{Z}$ every effective divisor on $X$ is cut out by a hypersurface in the ambient projective space, as assumed in Theorem A; (II) We extend Theorem A to meromorphic mappings from parabolic manifolds into $X \subset \mathbb{P}^{N}$. 
2. Divisors on projective variety $X$ with $\operatorname{Pic}(X)=\mathbb{Z}$. Theorem A (so the defect relation in Corollary $A$ ) only applies to those divisors on $X$ which are cut out by hypersurfaces in an ambient projective space. For a general non-singular projective variety, it is far from true that (multiples of) effective divisors are cut out by hypersurfaces in the ambient projective space. In this section we prove that, for a nonsingular projective variety $X$ with $\operatorname{Pic}(X)=\mathbb{Z}$, up to taking multiples, effective divisors are cut out by hypersurfaces. Therefore Theorem A in above can be applied.

Lemma 2.1. Let $X \subseteq \mathbb{P}^{N}$ be a nonsingular projective variety. Assume that $\operatorname{Pic}(X)=\mathbb{Z}$. Let $D$ be an effective divisor on $X$. Then there exists a positive integer $k$ such that $k D$ is the restriction to $X$ of some hypersurface in $\mathbb{P}^{N}$.

Proof. Consider the line bundle $\mathcal{O}_{X}(D)$, which represents an element in $\operatorname{Pic}(X)$. Due to $\operatorname{Pic}(X)=\mathbb{Z}$, there exist positive integers $m, \kappa$ such that $\mathcal{O}_{X}(m D)=\mathcal{O}_{X}(\kappa)$ as elements of $\operatorname{Pic}(X)$. We are free to multiply this equality with an arbitrary positive integer $\tilde{m}$, obtaining $\mathcal{O}_{X}(\tilde{m} m D)=\mathcal{O}_{X}(\tilde{m} \kappa)$. Consider the short exact sequence

$$
0 \rightarrow \mathcal{I}_{X}(\tilde{m} \kappa) \rightarrow \mathcal{O}_{\mathbb{P}^{N}}(\tilde{m} \kappa) \rightarrow \mathcal{O}_{X}(\tilde{m} \kappa) \rightarrow 0 .
$$

The corresponding long exact sequence starts with

$$
\begin{aligned}
0 & \rightarrow H^{0}\left(\mathbb{P}^{N}, \mathcal{I}_{X}(\tilde{m} \kappa)\right) \rightarrow H^{0}\left(\mathbb{P}^{N}, \mathcal{O}_{\mathbb{P}^{N}}(\tilde{m} \kappa)\right) \rightarrow H^{0}\left(X, \mathcal{O}_{X}(\tilde{m} \kappa)\right) \\
& \rightarrow H^{1}\left(\mathbb{P}^{N}, \mathcal{I}_{X}(\tilde{m} \kappa)\right) .
\end{aligned}
$$

Due to Serre's Vanishing Theorem, $H^{1}\left(\mathbb{P}^{N}, \mathcal{I}_{X}(\tilde{m} \kappa)\right)=0$ when $\tilde{m}$ is sufficiently large. Thus, all sections in $H^{0}\left(X, \mathcal{O}_{X}(\tilde{m} \kappa)\right)$ extend to sections in $H^{0}\left(\mathbb{P}^{N}, \mathcal{O}_{\mathbb{P}^{N}}(\tilde{m} \kappa)\right)$ when $\tilde{m}$ is sufficiently large. In particular, sections $s \in H^{0}\left(X, \mathcal{O}_{X}(\tilde{m} m D)\right)=H^{0}\left(X, \mathcal{O}_{X}(\tilde{m} \kappa)\right)$ with

$$
(s)=\tilde{m} m D
$$

extend. However, the sections of $\mathcal{O}_{\mathbb{P}^{N}}(\tilde{m} \kappa)$ correspond to homogeneous polynomials of degree $\tilde{m} \kappa$ in $\mathbb{C}\left[X_{0}, \ldots, X_{N}\right]$. Thus, $\tilde{m} m D$ is indeed defined by the restriction to $X$ of a homogeneous polynomial in $\mathbb{C}\left[X_{0}, \ldots, X_{N}\right]$ when $\tilde{m}$ is sufficiently large. This proves the lemma.

Let $X \subseteq \mathbb{P}^{N}$ be a nonsingular projective variety and let $L: \rightarrow X$ be an ample line bundle over $X$. Denote by $\|\cdot\|$ a Herimitian fiber metric on $L$ and $\omega$ be its Chern form. Let $f: \mathbb{C} \rightarrow X$ be a holomorphic mapping. We set

$$
T_{f}(r, L)=\int_{1}^{r} \frac{d t}{t} \int_{\{z|| z \mid<t\}} f^{*} \omega,
$$

and call it the characteristic function of $f$ with respect to $L$. It is easy to check that it is independent, up to a bounded term, of the choice of the Hermitian fiber metric on $L$. Let $D=(\sigma) \in|L|$ with $\|\sigma\|<1$ on $X$, where $|L|$ is the complete system of $L$. Assume that $f(\mathbb{C})$ is not contained in $\operatorname{Supp} D$. We define the approximity function of $f$ with respect to $D$ by

$$
m_{f}(r, D)=\int_{0}^{2 \pi} \log \left(\frac{1}{\left\|\sigma\left(f\left(r e^{i \theta}\right)\right)\right\|}\right) \frac{d \theta}{2 \pi} .
$$


To define the counting function for $D=(\sigma) \in|L|$, let $n_{f}(r, D)$ be the number of zeros of $\sigma \circ f$ in the disk $|z|<r$, counting multiplicity. The counting function is then defined by

$$
N_{f}(r, D)=\int_{1}^{r} \frac{n_{f}(t, D)}{t} d t .
$$

The Poincare-Lelong formula implies that (The First Main Theorem)

$$
T_{f}(r, L)=m_{f}(r, D)+N_{f}(r, D)+O(1)
$$

for all $D \in|L|$ with $f(\mathbb{C}) \not \subset S u p p(D)$, where $O(1)$ stands for a bounded term as $r \rightarrow+\infty$.

Let $f$ and $D$ be as above (with the assumption that $D$ is ample), we define Nevvanlinna's deficiency $\delta_{f}(D)$ by

$$
\delta_{f}(D)=\liminf _{r \rightarrow+\infty} \frac{m_{f}(r, D)}{T_{f}\left(r, \mathcal{O}_{X}(D)\right)}=1-\limsup _{r \rightarrow+\infty} \frac{N_{f}(r, D)}{T_{f}\left(r, \mathcal{O}_{X}(D)\right)},
$$

where $\mathcal{O}_{X}(D)$ is the line bundle over $X$ associated to the divisor $D$. Note that the Nevvanlinna's deficiency $\delta_{f}(D)$ only depends on $f$ and $D$ (independent of the choice of the metrics on $\mathcal{O}(D))$. We also note that for any integer positive integer $k, n_{f}(r, k D)=k n_{f}(r, D)$ and $T_{f}\left(r, \mathcal{O}_{X}(k D)\right)=k T_{f}\left(r, \mathcal{O}_{X}(D)\right)$, thus, for any integer positive integer $k$,

$$
\delta_{f}(k D)=\delta_{f}(D)
$$

This notion extends to any effective divisor $D$ since we can always write $D=D_{1}-D_{2}$ with $D_{1}, D_{2}$ being very ample.

The first result, among the two main results in this paper, is the following Theorem.

THEOREM 1 [Defect Relation]. Let $X$ be a non-singular projective variety of dimension $n$ with $\operatorname{Pic}(X)=\mathbb{Z}$. Let $D_{1}, \ldots, D_{q}$ be effective divisors on $X$ located in general position (i.e. for every subset $\left\{i_{0}, \ldots, i_{n}\right\} \subset\{1, \ldots, q\}$, supp $D_{i_{0}} \cap \cdots \cap$ supp $\left.D_{i_{n}}=\emptyset\right)$. Let $f: \mathbb{C} \rightarrow X$ be an algebraically nondegenerate holomorphic map. Then

$$
\sum_{j=1}^{q} \delta_{f}\left(D_{j}\right) \leq \operatorname{dim} X+1 .
$$

In particular, if $q \geq \operatorname{dim} X+2$, then every holomorphic map $f: \mathbb{C} \rightarrow X \backslash D$, where $D=\sum_{j=1}^{q} D_{j}$, must be algebraically degenerate, i.e., the image of $f$ must be contained in a proper subvariety of $X$.

Proof. By Lemma 2.1, there exist positive integers, say $k_{j}, 1 \leq j \leq q$, such that $k_{j} D_{j}$ are defined by the restriction to $X$ of homogeneous polynomials $Q_{j}$ in $\mathbb{C}\left[X_{0}, \ldots, X_{N}\right]$. From Corollary A, we get

$$
\sum_{j=1}^{q} \delta_{f}\left(k_{j} D_{j}\right) \leq \operatorname{dim} X+1 .
$$


But, as we noted in above,

$$
\delta_{f}\left(k_{j} D_{j}\right)=\delta_{f}\left(D_{j}\right)
$$

hence

$$
\sum_{j=1}^{q} \delta_{f}\left(D_{j}\right) \leq \operatorname{dim} X+1
$$

This proves Theorem 1 .

Next, we exhibit a large class of nonsingular projective varieties that satisfy the condition $\operatorname{Pic}(X)=\mathbb{Z}$, namely those of sufficiently small codimension. We refer the reader to [10] or [13, section 3.2] for more on the algebraic geometry behind this question, which is essentially the problem of extending the Lefschetz Hyperplane Theorem to nonsingular varieties that are not complete intersections. In particular, the theorems of [2], [12], and, in the case of a general ground field of characteristic zero, [14] apply. For our purposes, we simply state the following proposition, which is an immediate consequence of these theorems.

Proposition 2.1. Let $X \subset \mathbb{P}^{N}$ be a nonsingular projective variety of dimension $n$. If $2 n-N \geq 2$, then restriction yields an isomorphism

$$
\operatorname{Pic}\left(\mathbb{P}^{N}\right) \cong \operatorname{Pic}(X) .
$$

Since $\operatorname{Pic}\left(\mathbb{P}^{N}\right)=\mathbb{Z}$, the proposition gives the following immediate consequence of Corollary.

Corollary 2.1. Let $X \subseteq \mathbb{P}^{N}$ be a nonsingular projective variety. Assume that $2 \operatorname{dim} X-N \geq 2$ holds. Let $D=\sum_{j=1}^{q} D_{j}$ be an effective divisor on $X$ such that $D_{j}, 1 \leq j \leq q$, are in general position. Let $f: \mathbb{C} \rightarrow X$ be an algebraically nondegenerate holomorphic map. Then

$$
\sum_{j=1}^{q} \delta_{f}\left(D_{j}\right) \leq \operatorname{dim} X+1 .
$$

In particular, if $q \geq \operatorname{dim} X+2$, then every holomorphic map $f: \mathbb{C} \rightarrow X \backslash D$ must be algebraically degenerate, i.e., the image of $f$ must be contained in a proper subvariety of $X$.

We make a remark here about the nature of the condition $2 \operatorname{dim} X-N \geq 2$. It is well-known that, by general linear projections, any nonsingular projective variety of dimension $n$ can be embedded in $\mathbb{P}^{N}$ with $N=2 n+1$. Thus, for an arbitrary nonsingular projective variety, one can always find an embedding with $2 n-N=-1$. In particular, Corollary 2.1 applies to many interesting special projective varieties such as hypersurfaces, appropriate complete intersections, and certain Grassmannians, such as $G(2,5)$ embedded into $\mathbb{P}^{9}$ under the Plücker embedding. Note that the latter is not a complete intersection due to Bézout's Theorem, because its degree is (the prime number) 5 , while it is not contained in any hyperplane.

3. The second main theorem on parabolic manifolds. In this section, we extend Theorem A to meromorphic mappings from parabolic manifolds to algebraic variety $X \subset \mathbb{P}^{N}$. 
3.1. The result of Stoll-Wong. H. Cartan's defect relation mentioned in the introduction was also proved by L. Ahlfor's in 1941 (see [1]) with a different method. Ahlfors method was indeed more geometrical, which is a magnificent demonstration of the interplay between topology, geometry and analysis, on holomorphic curves. Ahlfors' theory was extended to parabolic Riemann surfaces by H. Weyl and J. Weyl in the famous monograph [25] published in 1943. Modern treatment of this theory can also be found in $\mathrm{Wu}[28]$. Extension of the theory to higher dimension was first achieved by Stoll in a series of papers [21], [22] in late forties and early fifties. In the sixties and early seventies, value distribution theory attracted the attention of many mathematicians, notably the works of Carlson-Griffiths [3], Griffiths-King [9] extending the theory to algebraic manifolds in the equi-dimensional case. However in this article we shall restrict ourselves to the ideas of Stoll in extending the defect relation to a larger case of manifolds other than $\mathbf{C}^{m}$.

In extending the defect relation, the class of manifolds should be large enough so as to include all affine algebraic manifolds and, on the other hand, manifolds in this class should not admit any non-constant bounded holomorphic functions (otherwise we cannot expect to have a nice simple defect relation). Such manifolds can be produced by the following procedure (cf. Stoll [21]). For simplicity we consider only the case of Kahler manifolds. Let $M$ be a complex manifold of dimension $m$ with Kahler metric $g$ and $\omega$ the associated Kahler form. Choose a sequence of relatively compact domains with smooth boundaries $\left\{G_{j}\right\}, G_{j} \subset \subset G_{j+1}$ and $\cup G_{j}=M$. Solve the Dirichlet problem for the Laplace-Beltrami operator $\triangle$ on $G_{j}-G_{0}$,

$$
\begin{gathered}
\triangle u_{j}=0 \text { on } G_{j}-G_{0} \\
u_{j}=\left\{\begin{array}{cc}
0 & \text { on } M-G_{j} \\
1 & \text { on } \overline{G_{0}}
\end{array} .\right.
\end{gathered}
$$

The harmonic condition (1.1) is equivalent to

$$
d d^{c} u_{j} \wedge \omega^{m-1}=0
$$

where $d^{c}=\sqrt{-1}(\bar{\partial}-\partial)$. Define constants

$$
C_{j}=-\int_{\partial G_{j}} d^{c} u_{j} \wedge \omega^{m-1} .
$$

These constants are strictly positive (by Hopf's lemma) and satisfies $C_{j} \geq C_{k}$ if $k \geq j$ (by maximum principle). Thus the limit

$$
C_{\infty}(M)=\lim _{j \rightarrow \infty} C_{j} \geq 0
$$

exists and is called the capacity of $M$ at infinity.

Proposition 3.1.1. A connected Kahler manifold $M$ with zero capacity at infinity does not admit any non-constant bounded holomorphic function.

Proof. Let $\omega$ be the fundamental Kahler form and suppose there is a bounded non-constant holomorphic function $f$, say $|f| \leq 1$. Then by (1.2) we have,

$$
0<\lambda=\int_{G_{0}} d d^{c} \log \left(1+|f|^{2}\right) \wedge \omega^{m-1}=\int_{G_{0}} u_{j} d d^{c} \log \left(1+|f|^{2}\right) \wedge \omega^{m-1} .
$$


From (1.1), (1.2) and Stoke's theorem, the last term above is equal to

$$
\int_{\partial G_{0}-\partial G_{j}} \log \left(1+|f|^{2}\right) d^{c} u_{j} \wedge \omega^{m-1} \leq-\int_{\partial G_{j}} \log \left(1+|f|^{2}\right) d^{c} u_{j} \wedge \omega^{m-1} \leq C_{j} \log 2 .
$$

Thus we have $0<\lambda \leq C_{j} \log 2$, for any $j$. This is a contradiction since $C_{j} \rightarrow 0$ as $j \rightarrow \infty$.

The concept of zero capacity above depends on the metric, thus it is desirable to have more intrinsic notion of "parabolicity". The following definition defining parabolicity via the homogeneous complex Monge-Ampere equation is due to Stoll (cf. [23]).

Definition 3.1.1. A continuous non-negative function $\tau$ on a complex manifold $M^{m}$ is parabolic if

(i) $\tau$ is $C^{\infty}$ on $M_{*}=M-\{\tau=0\}$;

(ii) $\tau$ is plurisubharmonic on $M$ and is strictly plurisubharmonic at some point of $M_{*}$;

(iii) the function $u=\log \tau$ satisfies the complex homogeneous Monge-Ampere equation on $M_{*}$, i.e.

$$
\operatorname{det}\left(u_{\alpha \bar{\beta}}\right) \equiv 0 \quad \text { on } M_{*}
$$

where $u_{\alpha \bar{\beta}}=\partial^{2} u / \partial z \partial \bar{z}$.

A complex manifold $M$ is parabolic if there exists a continuous exhaustion $\tau$ : $M \rightarrow[0, \infty)$ which is parabolic on the complement of some compact set $K$. Examples of parabolic manifolds include (1) Open parabolic Riemann surfaces (as in the classical sense); (2) Euclidean space $\mathbb{C}^{m}$ (with $\tau(z)=|z|^{2}$ ); (3) An affine algebraic manifold as it can be realized a finite branched cover $\pi: M \rightarrow \mathbb{C}^{m}$ (with $\left.\tau(z)=|\pi(z)|^{2}\right)$.

On a parabolic manifold $(M, \tau)$ take $r_{0}$ so that the set $M_{r_{0}}=\left\{\tau<r_{0}\right\}$ contains the exceptional compact set $K$. For any $r>r_{0}$, the function

$$
u_{r}=\left\{\begin{array}{cc}
0 & \text { on } M-M_{r} \\
\frac{\log r-\log \tau}{\log r-\log r_{0}} & \text { on } M_{r}-M_{r_{0}} \\
1 & \text { on } M_{r_{0}}
\end{array}\right.
$$

solves the Dirichelt problem (1.2) and (1.3) with $\omega=d d^{c} \log \tau$. Capacity can be defined as before. It is easy to verify that, since $\tau$ is unbounded, the capacity at infinity $C_{\infty}(M)$ vanishes. We thus have, from Proposition 3.1.1,

Corollary 3.1.1. Let $M$ be a parabolic manifold then $C_{\infty}(M)=0$ so it does not admit any non-constant bounded holomorphic function.

The following defect relation on parabolic manifolds is due to Stoll [23] and Wong $[26])$.

TheOREM 3.1.1 (Stoll-Wong). Let $(M, \tau)$ be a parabolic manifold and let $f: M \rightarrow$ $\mathbb{P}^{n}$ be a linearly non-degenerate meromorphic map. Let $H_{1}, \ldots, H_{q}$ be hyperplanes in general position in $\mathbb{P}^{n}$, then

$$
\sum_{j=1}^{q} \delta_{f}\left(H_{j}\right) \leq n+1+\frac{n(n+1)}{2} R_{f}+3 n(n+1) Y_{f}
$$


The term $R_{f}$, which vanishes in the case of $\mathbf{C}^{m}$, reflects the topology of the manifold $M$. The appearance of the term $Y_{f}$ (called the majorant defect) is due mainly to technicality. Due to the global nature of the theorem, it is necessary to define the derivatives (first and higher orders) of $f$ by a global (meromorphic) vector field $Z$ in such a way that $f \wedge Z f \wedge \cdots \wedge Z^{n} f \not \equiv 0$. The term $Y_{f}$ occurs in the estimate of the growth of $Z$, for which one does not have good control in general. A natural way of constructing $Z$ with "good" growth condition is to take the (complex) gradient vector field $Z$ of $\tau$. However, $Z$ is in general not holomorphic. If $Z$ is holomorphic, then the term $Y_{f}$ vanishes, this is the case for parabolic Riemann surfaces and for affine algebraic manifolds.

Corollary 3.1.2. If $M$ is algebraic, theorem 3.1.1 reduces to

(i) $\sum_{j=1}^{q} \delta_{f}\left(H_{j}\right) \leq n+1$ if $f$ is transcendental

(ii) $\sum_{j=1}^{q} \delta_{f}\left(H_{j}\right) \leq n+1+\frac{n(n+1)}{2} \frac{\operatorname{deg}(M)}{\operatorname{deg}(f)}$, if $f$ is rational.

3.2. Meromorphic mappings on $M$. Throughout the rest of the paper, we shall assume that $M$ is parabolic with the parabolic exhaustion $\tau$. Assume that $\operatorname{dim} M=m$. Take $r_{0}>1$ so that the set $M_{r_{0}}=\left\{\tau<r_{0}\right\}$ contains the exceptional compact set $K$. For $r_{0} \leq r \in \mathbb{R}$ and $A \subseteq M$ define

$$
\begin{array}{cc}
A[r]=\left\{x \in A \mid r_{0}<\tau(x) \leq r^{2}\right\}, & A(r)=\left\{x \in A \mid r_{0}<\tau(x)<r^{2}\right\}, \\
A\langle r\rangle=\left\{x \in A \mid \tau(x)=r^{2}\right\}, & A_{*}=\left\{x \in A \mid \tau(x)>r_{0}\right\}, \\
v=d d^{c} \tau, \quad \omega=d d^{c} \log \tau, \quad \sigma=d^{c} \log \tau \wedge \omega^{m-1} .
\end{array}
$$

Define

$$
\hat{\mathbb{R}}_{\tau}=\left\{r \in \mathbb{R}^{+} \mid d \tau(x) \neq 0 \quad \text { for all } \quad x \in M\langle r\rangle\right\} .
$$

Then $\mathbb{R}^{+} \backslash \hat{\mathbb{R}}_{\tau}$ has measure zero. If $r \in \hat{\mathbb{R}}_{\tau}$, the boundary $\partial M(r)=M\langle r\rangle$ is a compact, real, $(2 m-1)$-dimensional submanifold of class $C^{\infty}$ of $M$, oriented to the exterior of $M\langle r\rangle$. By Stoll ([23], p. 133), for all $r \in \hat{\mathbb{R}}_{\tau}$,

$$
\varsigma:=\int_{M\langle r\rangle} \sigma
$$

is a positive constant, independent of $r$. From now on, we always assume that $r>r_{0}$ and $r \in \hat{\mathbb{R}}_{\tau}$.

Let $A \neq \emptyset$ be a nonempty open subset of $M$ such that $S=M-A$ is analytic. Let $f: M \rightarrow \mathbb{P}^{n}(\mathbb{C})$ be holomorphic on $A$. The closure $\Gamma$ of the graph $\{(x, f(x)) \mid x \in A\}$ in $M \times \mathbb{P}^{n}(\mathbb{C})$ is called the closed graph of $f$. The map $f$ is said to be meromorphic on $M$ if (i) $\Gamma$ is analytic in $M \times \mathbb{P}^{n}(\mathbb{C})$ and (ii) $\Gamma(f) \cap\left(K \times \mathbb{P}^{n}(\mathbb{C})\right)$ is compact for each compact subset $K \subseteq M$, i.e. the projection $\rho: \Gamma(f) \rightarrow M$ is proper. If $f$ is meromorphic, then the set of indeterminacy $I_{f}=\left\{x \in M \mid \# \rho^{-1}(x)>1\right\}$ is analytic with $\operatorname{dim} I_{f} \leq m-2$ 
and is contained in $S$. $f$ extends holomorphically to $f: M-I_{f} \rightarrow \mathbb{P}^{n}(\mathbb{C})$ such that we can assume, a posteriori, that $S=I_{f}$. If $m=1, I_{f}$ is necessarily empty and $f: M \rightarrow \mathbb{P}^{n}(\mathbb{C})$ is holomorphic. Given $M, A, S$ and a holomorphic map $f: A \rightarrow \mathbb{P}^{n}(\mathbb{C})$ as above. A holomorphic map $\mathbf{f}(\not \equiv 0): U \rightarrow \mathbb{C}^{n+1}$ on an open and connected subset $U$ of $M$ is said to be a representation of $f$ if $f(x)=\mathbb{P}(\mathbf{f}(x))$ for all $x \in A \cap U$ with $\mathbf{f}(x) \neq 0$. A representation $\mathbf{f}$ is said to be reduced if $\operatorname{dim} \mathbf{f}^{-1}(0) \leq m-2$. The map $f$ is meromorphic if and only if for every point $p \in M$, there is a representation $\mathbf{f}: U \rightarrow \mathbb{C}^{n+1}$ of $f$ with $p \in U$. If so, a representation $\mathbf{f}$ is reduced if and only if $U \cap I_{f}=\mathbf{f}^{-1}(0)$. There is also a reduced representation at every point $p \in M$.

3.3. The associated maps. To define the associated maps of $f$, we need to assume that there exists a holomorphic form $B$ of bidegree $(m-1,0)$ on $M$. Let $\mathbf{f}$ be a holomorphic vector-valued function on an open subset $U$ of $M$. If $z=\left(z_{1}, \ldots, z_{m}\right)$ is a chart with $U_{z} \cap U \neq \emptyset$, then the $B$-derivative $\mathbf{f}_{B, z}^{\prime}=\mathbf{f}^{\prime}$ on $U \cap U_{z}$ for $z$ is defined by $d \mathbf{f} \wedge B=\mathbf{f}^{\prime} d z_{1} \wedge \cdots \wedge d z_{m}$. The operation can be iterated so that the $k$-th $B$-derivative $\mathbf{f}^{(k)}$ is defined: $\mathbf{f}^{(k)}=\left(\mathbf{f}^{(k-1)}\right)^{\prime}$. Put $\mathbf{f}^{(0)}=\mathbf{f}$. Abbreviate

$$
\mathbf{f}_{k}=\mathbf{f} \wedge \mathbf{f}^{\prime} \wedge \cdots \wedge \mathbf{f}^{(k)}: U \rightarrow \wedge^{k+1} \mathbb{C}^{n+1} .
$$

Let $f: M \rightarrow \mathbb{P}^{n}(\mathbb{C})$ be a meromorphic map. If $\mathbf{f}_{k} \neq \equiv 0$ for one choice of a reduced representation $\mathbf{f}: U \rightarrow \mathbb{C}^{n+1}$ on a chart $U_{z}$, then $\mathbf{f}_{k} \not \equiv 0$ for all possible choices and $f$ is said to be general of order $k$ for $B$. In this case, the $k$-th associated map $f_{k}: M \rightarrow \mathbb{P}\left(\bigwedge^{k+1} \mathbb{C}^{n+1}\right)$ is well-defined as a meromorphic map by $f_{k} \mid U=\mathbb{P}\left(\mathbf{f}_{k}\right)$ for all possible choices of $\mathbf{f}$ and chart $z$. We say that $f$ is general for $B$ if $f$ is general of order $k$ for $B$ for all $k, 1 \leq k \leq n$. The basic existence theorem for a holomorphic $(m-1)$ form $B$ on $M$ is due to W. Stoll. He (see [23]) proved the following statement: Let $M$ be a connected Stein manifold and let $f: M \rightarrow \mathbb{P}^{n}(\mathbb{C})$ be a linearly nondegenerate meromorphic map. Then there exists a holomorphic $(m-1)$-form $B$ on $M$ such that $f$ is general for $B$. If $\operatorname{dim} M=1$, we may take $B \equiv 1$. If $M$ is affine algebraic with the exhaustion $\tau$ defined as above and $\operatorname{dim} M \geq 2$, then the form $B$ can be chosen so that

$$
m i_{m-1} B \wedge \bar{B} \leq(1+\tau)^{n-1}\left(d d^{c} \tau\right)^{m-1}
$$

where $i_{m-1}=\left(\frac{\sqrt{-1}}{2 \pi}\right)(m-1) !(-1)^{(m-1)(m-2) / 2}$.

3.4. The majorant function. For a general parabolic Stein manifold $(M, \tau)$, even though the existence of $B$ exists by Stoll's result above, in general, it may not have a polynomial type estimate as for affine algebraic manifolds. To overcome this difficulty, Stoll [23] postulates the existence of a majorant function such that

$$
m i_{m-1} B \wedge \bar{B} \leq Y(r) v^{m-1}
$$

on $M[r]$. Such function $Y(r)$ is called a majorant for $B$. The majorant function $Y(r)$ introduces an extra term in the Second Main Theorem.

3.5. The Plücker formula. Let $f: M \rightarrow \mathbb{P}^{n}(\mathbb{C})$ be a meromorphic map which is linearly non-degenerate, hence $f$ is general for $B$. Let $f_{k}$ be the $k$ th associated map of $f$. Let $\Omega_{k}$ be the Fubini-Study form on $\mathbb{P}^{n}\left(\bigwedge^{k+1} \mathbb{C}^{n+1}\right)$. Then we have (see [23], or [27] Lemma 1.10)

$$
m i_{m-1} f_{k}^{*}\left(\Omega_{k}\right) B \wedge \bar{B}=\frac{\left\|\mathbf{f}_{k-1}\right\|\left\|\mathbf{f}_{k+1}\right\|}{\left\|\mathbf{f}_{k-1}\right\|} m ! d z_{1} \wedge d \bar{z}_{1} \wedge \cdots \wedge d z_{m} \wedge d \bar{z}_{m} .
$$


Define the $k$ th characteristic function

$$
T_{f_{k}}(r)=\int_{r_{0}}^{r} \frac{d t}{t^{2 m-1}} \int_{M[t]} f_{k}^{*}\left(\Omega_{k}\right) \wedge v^{m-1} .
$$

It is known that $T_{f_{n}}(r) \equiv 0$. Denote $T_{f_{-1}}(r) \equiv 0$.

Let $\nu$ be a divisor on $M$ with $S=\operatorname{supp} \nu$. The counting function of $\nu$ is defined to be

$$
N_{\nu}(r)=\int_{r_{0}}^{r} n_{\nu}(t) \frac{d t}{t}
$$

where

$$
\begin{gathered}
n_{\nu}(t)=t^{2-2 m} \int_{S[t]} \nu v^{m-1}=\int_{S_{*}[t]} \nu \omega^{m-1}+n_{\nu}(0), \quad \text { if } m>1, \\
n_{\nu}(t)=\sum_{z \in S[t]} \nu(z), \quad \text { if } m=1 .
\end{gathered}
$$

Let $d_{k}$ be the zero divisor of $f_{k}$ ( when $k=n$, we obtain the Wronskian divisor $d_{n}$ ). The divisor $l_{k}=d_{k-1}-2 d_{k}+d_{k+1} \geq 0$ is called the $k$ th stationary index, here we assume that $d_{-1}=0$. Let $I_{k}$ be the indeterminacy of $f_{k}$. On $M-I_{k}$ define,

$$
h_{k}^{2}:=\frac{m i_{m-1} f_{k}^{*}\left(\Omega_{k}\right) \wedge B \wedge \bar{B}}{v^{m}} .
$$

and define

$$
S_{k}(r)=\int_{M\langle r\rangle} \log h_{k}^{2} \sigma
$$

Theorem 3.5.1 [Plücker Formula] ([23], Theorem 7.6]).

$$
N_{l_{k}}(r)+T_{f_{k-1}}(r)-2 T_{f_{k}}(r)+T_{f_{k+1}}(r)=S_{k}(r)-S_{k}\left(r_{0}\right)+R i c_{\tau}(r) .
$$

The function $\operatorname{Ric}_{\tau}(r)$ is called the Ricci function (c.f. [23], or see (3.2) in $[27]$ ). It depends only on the geometry (topology) of the manifold M. The other terms of Plucker Formula are analytic invariants. The Ricci function is the integrated Euler characteristic. For affine algebraic manifolds $\pi: M \rightarrow \mathbb{C}^{m}$, we have $\lim _{r \rightarrow+\infty} \frac{\operatorname{Ric}_{\tau}(r)}{\log r}=d_{\pi}=$ degree of the branching divisor of $\pi$.

3.6. Projective distance. Denote by $\mathbb{C}^{* n+1}$ the dual space of $\mathbb{C}^{n+1}$. For $0 \leq$ $k \leq n$, let $\left\lfloor:\left(\bigwedge^{k+1} \mathbb{C}^{n+1}\right) \times \mathbb{C}^{* n+1} \rightarrow \bigwedge^{k} \mathbb{C}^{n+1}\right.$ be the interior product defined in the usual way. Let $x \in \mathbb{P}\left(\bigwedge^{k+1} \mathbb{C}^{n+1}\right)$ with representative $\xi \in \bigwedge^{k+1} \mathbb{C}^{n+1}-\{0\}$ and let $a \in \mathbb{P}\left(\mathbb{C}^{* n+1}\right)$ with representative $\alpha \in \mathbb{C}^{* n+1}-\{0\}$, the projective distance between $x$ and $a$ is defined by

$$
0 \leq\|x ; a\|=\frac{\| \xi\lfloor\alpha \|}{\|\xi\|\|\alpha\|} \leq 1
$$


where the norm on on $\bigwedge^{k} \mathbb{C}^{n+1}$ is induced by the standard norm on $\mathbb{C}^{n+1}$. Note that the above definition is independent of choice of the representatives $\alpha$ and $\xi$. Note that a hyperplane $H$ in $\mathbb{P}^{n}(\mathbb{C})$ can also be regarded as a point in $\mathbb{P}^{n}\left(\mathbb{C}^{*}\right)$. Hence, for every meromorphic map $f: M \rightarrow \mathbb{P}^{n}(\mathbb{C}),\left\|f_{k}(z) ; H\right\|$ is defined for $z \in M$. This gives a distance function (from $f_{k}(z)$ to $H$ ) on $M$. For a hyperplane $H$ in $\mathbb{P}^{n}(\mathbb{C})$, define the proximity function

$$
m_{f_{k}}(r, H)=\int_{M\langle r\rangle} \log \frac{1}{\left\|f_{k} ; H\right\|} \sigma .
$$

For a hyperplane $H$ in $\mathbb{P}^{n}(\mathbb{C})$, define an $H$-divisor $\nu=\mu_{f_{k}}^{H}$ as in Stoll [23] let $N_{f_{k}}(r, H)=N_{\nu}(r)$ as defined in (1.7).

Theorem 3.6.1 [First Main Theorem] ([23], (8.21), P.153). Let $f: M \rightarrow \mathbb{P}^{n}(\mathbb{C})$ be a meromorphic map which is general for $B$. Then, for every hyperplane $H \in \mathbb{P}^{n}(\mathbb{C})$ and for every $0 \leq k \leq n$, we have

$$
T_{f_{k}}(r) \geq N_{f_{k}}(r, H)+m_{f_{k}}(r, H)-m_{f_{k}}\left(r_{0}, H\right) .
$$

Similarly, let $D$ be a hypersurface in $\mathbb{P}^{n}(\mathbb{C})$ of degree $d$. Assume that $f(M) \not \subset D$. Let $Q$ be the homogeneous polynomial (form) of degree $d$ defining $D$. The proximity function $m_{f}(r, D)$ is defined as,

$$
m_{f}(r, D)=\int_{M\langle r\rangle} \log \frac{\|\mathbf{f}(z)\|^{d}\|Q\|}{|Q(\mathbf{f})(z)|} \sigma .
$$

Let $N_{f}(r, D)=N_{\nu}(r)$, with $\nu=\mu_{Q(\mathbf{f})}$ as defined in (1.7).

Theorem 3.6.2 [First Main Theorem]. Let $f: M \rightarrow \mathbb{P}^{n}(\mathbb{C})$ be a holomorphic map, and let $D$ be a hypersurface in $\mathbb{P}^{n}(\mathbb{C})$ of degree d. If $f(M) \not \subset D$, then

$$
m_{f}(r, D)+N_{f}(r, D)=d T_{f}(r)+O(1)
$$

where $O(1)$ is a constant independent of $r$.

3.7. The Ahlfors' Estimate. We first recall the following theorem.

Theorem 3.7.1 [Ahlfors Estimate] ([23], Theorem 10.3). Let $H$ be a hyperplane in $\mathbb{P}^{n}(\mathbb{C})$. Then for any $0<\lambda<1$, we have

$$
\int_{r_{0}}^{r} \frac{d t}{t^{2 m-1}} \int_{M[t]} \frac{\left\|f_{k+1} ; H\right\|^{2}}{\left\|f_{k} ; H\right\|^{2-2 \lambda}} h_{k}^{2} v^{m} \leq \frac{8 Y(r)}{\lambda^{2}}\left(\lambda T_{f_{k}}(r)+\varsigma\right),
$$

where $\varsigma$ is defined in (1.4).

To re-formulate Theorem 3.7.1 to meet our purpose, we recall the following Calculus lemma.

Lemma 3.7.1 (Calculus Lemma). Let $T(r) \geq 0\left(r \geq r_{0} \geq 0\right)$ be a monotone increasing function. For an arbitrary $\epsilon>0$

$$
\frac{d}{d r} T(r) \leq T(r) \cdot \log ^{1+\epsilon} T(r) \|_{E(\epsilon)}
$$


where $\|_{E(\epsilon)}$ means that the inequality holds for all $r$ except a set $E_{\epsilon}$ (depends on $\epsilon$ ) with finite Lebesgue measure.

Proof. Since $T(r)$ is monotone increasing, the derivative $T^{\prime}(r)$ exists almost everywhere. We may assume that $T(r) \not \equiv 0$. Set

$$
E(\epsilon)=\left\{r \geq r_{0} \mid T^{\prime}(r) \geq T(r) \cdot \log ^{1+\epsilon} T(r)\right\}
$$

Then

$$
\operatorname{meas}(E(\epsilon))=\int_{E(\epsilon)} d r \leq \int_{r_{1}}^{\infty} \frac{T^{\prime}(r)}{T(r) \cdot \log ^{1+\epsilon} T(r)} d r<+\infty
$$

which proves the lemma.

The typical use of the calculus lemma is as follows:

LEMMA 3.7.2. Let $h$ be a nonnegative measurable function on $M$ such that $h v^{m}$ is locally integrable. Let $T$ be a function defined by

$$
T(r)=\int_{r_{0}}^{r} \frac{d t}{t^{2 m-1}} \int_{M[t]} h v^{m}
$$

Then, for an arbitrary $\epsilon>0$,

$$
\int_{M\langle r\rangle} h \sigma \leq T(r) \log ^{1+\epsilon} T(r)\left(\log \left(r^{2 m-1} T(r) \log ^{1+\epsilon} T(r)\right)\right)^{1+\epsilon} \|_{E(\epsilon)} .
$$

Proof. Notice that

$$
\int_{M[r]} h v^{m}=m \int_{M[r]} h \tau^{m-1} d \tau \wedge \sigma=2 m \int_{0}^{r}\left(\int_{M<r>} h \sigma\right) t^{2 m-1} d t .
$$

Hence

$$
2 m \int_{M\langle r\rangle} h \sigma=r^{-(2 m-1)} \frac{d}{d r}\left(r^{2 m-1} \frac{d T}{d r}\right) .
$$

Thus may apply the Calculus lemma twice, first to the function $r^{2 m-1} T^{\prime}(r)$ and then to the function $T^{\prime}(r)$. This proves the result.

Lemma 3.7.3. Let $S_{k}(r)$ be defined in (1.9). Then

$$
S_{k}(r) \leq \varsigma\left(\log ^{+} T_{f_{k}}(r)+\log ^{+} Y(r)+2 \log ^{+} \log r\right) \|_{E}
$$

where $E \subset\left[r_{0},+\infty\right)$ is a set with finite measure, and $\varsigma$ is given in (1.4).

Proof. We wish to applying Lemma 3.7.2 to $h_{k}^{2}$ which is defined in (1.8). To do so, we first notice

$$
\begin{aligned}
& \int_{r_{0}}^{r} \frac{d t}{t^{2 m-1}} \int_{M[t]} h_{k}^{2} v^{m}=m i_{m-1} \int_{M[t]} f_{k}^{*} \Omega \wedge B \wedge \bar{B} \\
& \leq Y(r) \int_{r_{0}}^{r} \frac{d t}{t^{2 m-1}} \int_{M[t]} f_{k}^{*} \Omega \wedge v^{m-1}=Y(r) T_{f_{k}}(r) .
\end{aligned}
$$


Hence, Lemma 3.7.2 with $\epsilon=1$

$$
\int_{M\langle r\rangle} h_{k}^{2} \sigma \leq Y(r) T_{f_{k}}(r)\left[\log \left(Y(r) T_{f_{k}}(r)\right)\right]^{2}\left\{\log \left[r^{2 m-1} Y(r) T_{f_{k}}(r) \log ^{2}\left(Y(r) T_{f_{k}}(r)\right)\right]\right\}^{2} \|_{E}
$$

where $E \subset\left[r_{0},+\infty\right)$ is a set with finite measure. Hence

$$
\begin{aligned}
S_{k}(r)= & \frac{\varsigma}{2} \int_{M\langle r\rangle} \log h_{k}^{2} \frac{\sigma}{\varsigma} \leq \frac{\varsigma}{2} \log \int_{M\langle r\rangle} h_{k}^{2} \frac{\sigma}{\varsigma} \\
\leq & \frac{\varsigma}{2}\left[\log ^{+} T_{f_{k}}(r)+\log ^{+} Y(r)+4 \log ^{+} \log T_{f_{k}}(r)+4 \log ^{+} \log ^{+} Y(r)+2 \log ^{+} \log r\right. \\
& \left.+2 \log ^{+} \log ^{+} \log ^{+} Y(r)+2 \log ^{+} \log ^{+} \log ^{+} T_{f_{k}}(r)+O(1)\right] \|_{E} .
\end{aligned}
$$

By enlarging $E$ if necessary, we can assume that $\left.4 \log ^{+} \log ^{+} \log T_{f_{k}}(r)\right) \leq$ $\log ^{+} T_{f_{k}}(r)\left\|_{E}, 8 \log ^{+} \log Y(r) \leq \log T_{f_{k}}(r)\right\|_{E}, 4 \log ^{+} \log ^{+} \log Y(r) \leq \log ^{+} Y(r) \|_{E}$, $8 \log ^{+} \log Y(r) \leq \log ^{+} Y(r) \|_{E}$ and $O(1) \leq \log ^{+} \log r \|_{E}$. Thus we get

$$
S_{k}(r) \leq \varsigma\left(\log ^{+} Y(r)+\log ^{+} T_{f_{k}}(r)+2 \log ^{+} \log r\right) \|_{E} .
$$

This proves the Lemma.

Theorem 3.7.2. For $0 \leq k \leq n-1$,

$$
T_{f_{k}}(r) \leq 2(k+1) T_{f}(r)+k(k+1) R i c_{\tau}(r)+k(k+1) \varsigma\left[\log ^{+} Y(r)+2 \log ^{+} \log r\right] \|_{E} .
$$

where $E \subset\left(r_{0}, \infty\right)$ is a set with finite measure.

Proof. Summering Theorem 3.5.1 (the Plucker formula) from $p=0$ to $p=k$, we get

$$
T_{f_{k}}(r)-(k+1) T_{0}(r) \leq \frac{k(k+1)}{2} R i c_{\tau}(r)+\sum_{0 \leq p \leq k-1}(k-p) S_{p}(r),
$$

where $T_{0}(r)=T_{f}(r)$. This, together with Lemma 3.7.3, yields

$$
\begin{aligned}
& T_{f_{k}}(r) \leq(k+1) T_{f}(r)+\frac{k(k+1)}{2} \operatorname{Ric}_{\tau}(r) \\
& +\frac{k(k+1)}{2} \varsigma\left[\log ^{+} Y(r)+\log ^{+} T(r)+2 \log ^{+} \log r\right] \|_{E}
\end{aligned}
$$

where $T(r)=\max _{0 \leq p \leq k} T_{f_{p}}(r)$. Notice that above inequality holds for all $k$ with $k=0,1, \ldots, n-1$, hence

$$
\begin{aligned}
T(r)= & \max _{0 \leq p \leq k} T_{f_{p}}(r) \leq(k+1) T_{f}(r)+\frac{k(k+1)}{2} \operatorname{Ric}_{\tau}(r) \\
& +\frac{k(k+1)}{2} \varsigma\left[\log ^{+} Y(r)+\log ^{+} T(r)+2 \log ^{+} \log r\right] \|_{E} .
\end{aligned}
$$

Using $\frac{k(k+1)}{2} \varsigma \log ^{+} T(r) \leq \frac{1}{2} T(r) \|_{E}$ for some $E \subset\left(r_{0}, \infty\right)$ with finite measure since $f$ is linearly non-degenerate, we derive

$$
T(r) \leq 2(k+1) T_{f}(r)+k(k+1) \operatorname{Ric}_{\tau}(r)+k(k+1) \varsigma\left[\log ^{+} Y(r)+2 \log ^{+} \log r\right] \|_{E} .
$$

Noticing that $T_{f_{k}}(r) \leq T(r)$, the above implies that the inequality in Theorem 3.7.2 holds. 
Theorem 3.7.3 (Ahlfors Estimate II). Let $H$ be a hyperplane in $\mathbb{P}^{n}(\mathbb{C})$. Let $\Lambda(r)=\min _{k}\left\{1 /\left(1+T_{f_{k}}(r)\right)\right\}$. Then, for every $0 \leq k \leq n-1$,

$$
\begin{aligned}
& \log ^{+} \int_{M\langle r\rangle}\left(\frac{\left\|f_{k+1} ; H\right\|^{2}}{\left\|f_{k} ; H\right\|^{2-2 \Lambda(r)}} h_{k}^{2}\right) \sigma \\
\leq & 2 \log ^{+} T_{f}(r)+2 \log ^{+} \operatorname{Ric}_{\tau}(r)+\log ^{+} Y(r)+10 \log ^{+} \log T_{f}(r)+9 \log ^{+} \log ^{+} \operatorname{Ric}_{\tau}(r) \\
& 10 \log ^{+} \log ^{+} Y(r)+4 \log ^{+} \log r \|_{E} .
\end{aligned}
$$

where $E \subset\left[r_{0},+\infty\right)$ is a set with finite measure.

Proof. Define functions

$$
K_{k}(r)=\int_{r_{0}}^{r} \frac{d t}{t^{2 m-1}} \int_{M[t]} \frac{\left\|f_{k+1} ; H\right\|^{2}}{\left\|f_{k} ; H\right\|^{2-2 \Lambda^{*}}} h_{k}^{2} v^{m}
$$

where $\Lambda^{*}=\Lambda \circ \tau^{1 / 2}$. By Lemma 3.7 .2 with $\epsilon=1$, we have

$$
\int_{M\langle r\rangle} \frac{\left\|f_{k+1} ; H\right\|^{2}}{\left\|f_{k} ; H\right\|^{2-2 \Lambda(r)}} h_{k}^{2} \sigma \leq K_{k}(r) \log ^{2} K_{k}(r)\left[\log \left(r^{2 m-1} K_{k}(r) \log ^{2} K_{k}(r)\right)\right]^{2} \|_{E} .
$$

On the other hand, noticing that $\Lambda$ is a decreasing function, we have $\left\|f_{k} ; H\right\|^{\Lambda^{*}} \leq$ $\left\|f_{k} ; H\right\|^{\Lambda(r)}$. Hence by Ahlfors' estimate with $\lambda=\Lambda(r)$, we have

$$
\begin{aligned}
K_{k}(r) & =\int_{r_{0}}^{r} \frac{d t}{t^{2 m-1}} \int_{M[t]} \frac{\left\|f_{k+1} ; H\right\|^{2}}{\left\|f_{k} ; H\right\|^{2-2 \Lambda^{*}}} h_{k}^{2} v^{m} \\
& \leq \frac{8 Y(r)}{\Lambda^{2}(r)}\left(\Lambda(r) T_{f_{k}}(r)+\varsigma\right) \\
& \leq 8 Y(r)\left(1+T_{f_{k}}(r)\right)^{2}\left(\frac{T_{f_{k}}(r)}{1+T_{f_{k}}(r)}+\varsigma\right) \\
& \leq 9(1+\varsigma) T_{k}^{2}(r) Y(r) .
\end{aligned}
$$

Combining (1.10) and (1.11),

$$
\begin{aligned}
& \log ^{+} \int_{M\langle r\rangle} \frac{\left\|f_{k+1} ; H\right\|^{2}}{\left\|f_{k} ; H\right\|^{2-2 \Lambda(r)}} h_{k}^{2} \sigma \\
\leq & 2 \log ^{+} T_{f_{k}}(r)+\log ^{+} Y(r)+8 \log ^{+} \log ^{+} T_{f_{k}}(r)+4 \log ^{+} \log ^{+} Y(r) \\
& +2 \log ^{+} \log ^{+} \log Y(r)+2 \log ^{+} \log ^{+} \log ^{+} T_{f_{k}}(r)+2 \log ^{+} \log r+O(1) \|_{E} .
\end{aligned}
$$

By enlarge $E$ if necessary, we can assume that $2 \log ^{+} \log ^{+} \log ^{+} T_{f_{k}}(r) \leq$ $\log ^{+} \log T_{f_{k}}(r)\left\|_{E}, \quad 2 \log ^{+} \log ^{+} \log ^{+} Y(r) \leq \log ^{+} \log ^{+} Y(r)\right\|_{E}$ and $O(1) \leq$ $\log ^{+} \log r \|_{E}$. Thus we get

$$
\begin{aligned}
& \log ^{+} \int_{M\langle r\rangle} \frac{\left\|f_{k+1} ; H\right\|^{2}}{\left\|f_{k} ; H\right\|^{2-2 \Lambda(r)}} h_{k}^{2} \sigma \\
\leq & 2 \log ^{+} T_{f_{k}}(r)+\log ^{+} Y(r)+9 \log ^{+} \log ^{+} T_{f_{k}}(r)+5 \log ^{+} \log ^{+} Y(r)+3 \log ^{+} \log r \|_{E} .
\end{aligned}
$$


Now use Theorem 3.7.2, the above inequality becomes

$$
\begin{aligned}
& \log ^{+} \int_{M\langle r\rangle} \frac{\left\|f_{k+1} ; H\right\|^{2}}{\left\|f_{k} ; H\right\|^{2-2 \Lambda(r)}} h_{k}^{2} \sigma \\
\leq & 2 \log ^{+} T_{f}(r)+2 \log ^{+} \operatorname{Ric}_{\tau}(r)+\log ^{+} Y(r)+9 \log ^{+} \log ^{+} T_{f}(r)+7 \log ^{+} \log Y(r) \\
& +9 \log ^{+} \log ^{+} \operatorname{Ric}_{\tau}(r)+9 \log ^{+} \log ^{+} \log ^{+} Y(r)+9 \log ^{+} \log ^{+} \log r \\
& +2 \log ^{+} \log r+O(1) \|_{E} .
\end{aligned}
$$

Again, by enlarge $E$ if necessary, we can assume that $9 \log ^{+} \log ^{+} \log Y(r) \leq$ $\log ^{+} \log ^{+} Y(r)\left\|_{E}, 9 \log ^{+} \log ^{+} \log r \leq \log ^{+} \log r\right\|_{E}$, and $O(1) \leq \log ^{+} \log r \|_{E}$. Hence

$$
\begin{aligned}
& \log ^{+} \int_{M\langle r\rangle} \frac{\left\|f_{k+1} ; H\right\|^{2}}{\left\|f_{k} ; H\right\|^{2-2 \Lambda(r)}} h_{k}^{2} \sigma \\
\leq & 2 \log ^{+} T_{f}(r)+2 \log ^{+} \operatorname{Ric}_{\tau}(r)+\log ^{+} Y(r)+10 \log ^{+} \log T_{f}(r) \\
& +9 \log ^{+} \log ^{+} \operatorname{Ric}_{\tau}(r)+10 \log ^{+} \log ^{+} Y(r)+4 \log ^{+} \log r \|_{E} .
\end{aligned}
$$

This concludes the proof.

3.8. A slight generalization of Wong-Stoll's theorem. In this subsection, we extend the Second Main Theorem of Wong-Stoll to the case where the given hyperplanes $H_{1}, \ldots, H_{q}$ in $\mathbb{P}^{n}(\mathbb{C})$ are not necessarily in general position.

To establish the value distribution theory on parabolic manifolds $M$, similar to [23], we make the following assumptions on $M$ (c.f. subsection 3.1-3.4): (i) $M$ is a connected complex manifold of dimension $m$; (ii) there exists a parabolic exhaustion function $\tau$ on $M$; (iii) For every integer $n$ and every linearly non-degenerate map $f: M \rightarrow \mathbb{P}^{n}(\mathbb{C})$, there is a holomorphic differential form $B$ of degree $(m-1,0)$ on $M$ such that $f$ is general for $B$ and

$$
m i_{m-1} B \wedge \bar{B} \leq Y(r) v^{m-1}
$$

on $M[r]$ for some real positive valued function $Y(r)$ on $M$, which is independent of $f$ ( $Y$ is called a majorant for $B$ ) where, for any positive integer $m$,

$$
i_{m}=\left(\frac{\sqrt{-1}}{2 \pi}\right)^{m}(-1)^{\frac{m(m-1)}{2}} m ! .
$$

A complex manifold $M$ with the assumptions (i)-(iii) is called an admissible parabolic manifold. Throughout this paper, we shall work on admissible parabolic manifolds.

THEOREM 3.8.1. Let $M$ be an admissible parabolic manifold of complex dimension $m$. Let $f: M \rightarrow \mathbb{P}^{n}(\mathbb{C})$ be a meromorphic map which is linearly non-degenerate. Let $\epsilon>0$ and let $H_{1}, \ldots, H_{q}$ be arbitrary hyperplanes in $\mathbb{P}^{n}(\mathbb{C})$. Then

$$
\begin{aligned}
& \int_{M\langle r\rangle} \max _{K} \sum_{j \in K} \log \frac{1}{\left\|f(z) ; H_{j}\right\|} \sigma \leq(n+1) T_{f}(r)+\frac{n(n+1)}{2} \operatorname{Ric}_{\tau}\left(r, s_{0}\right) \\
& -N_{d_{n}}\left(r, s_{0}\right)+\varsigma \frac{n(n+1)}{2}\left[\log ^{+} T_{f}(r)+\log ^{+} \operatorname{Ric}_{\tau}(r)+\frac{1}{2} \log ^{+} Y(r)\right. \\
& \left.+5 \log ^{+} \log T_{f}(r)+5 \log ^{+} \log ^{+} \operatorname{Ric}_{\tau}(r)+5 \log ^{+} \log ^{+} Y(r)+3 \log ^{+} \log ^{+} r\right] \|_{E}
\end{aligned}
$$


where $E \subset\left[r_{0},+\infty\right)$ is a set with finite measure, and the max is taken over all subsets $K$ of $\{1, \ldots, q\}$ such that the linear forms $H_{j}, j \in K$, are linearly independent.

Proof. Denote by $K \subset\{1, \ldots, q\}$ such that linear forms $\left\{H_{k}, k \in K\right\}$, are linearly independent. Without loss of generality, we may assume $q \geq n+1$ and that $\# K=n+1$. Let $T$ be the set of all the injective maps $\mu:\{0,1, \ldots, n\} \rightarrow$ $\{1, \ldots, q\}$ such that $H_{\mu(0)}, \ldots, H_{\mu(n)}$ are linearly independent. Denote by $\Gamma=$ $\max _{1 \leq j \leq q}\left\{\sum_{k=0}^{n-1} m_{f_{k}}\left(s_{0}, H_{j}\right)\right\}$ and $\Lambda(r)=\min _{k}\left\{1 /\left(1+T_{f_{k}}(r)\right)\right\}$. For any $\mu \in T, z \notin$ $I_{f}$, the Product to Sum Estimate (see [27] Lemma 1.12), with $\lambda=\Lambda(r)$, reads

$$
\prod_{j=0}^{n} \frac{\left\|f_{k+1}(z) ; H_{\mu(j)}\right\|^{2}}{\left\|f_{k}(z) ; H_{\mu(j)}\right\|^{2-2 \Lambda(r)}} \leq c_{k}\left(\sum_{j=0}^{n} \frac{\left\|f_{k+1}(z) ; H_{\mu(j)}\right\|^{2}}{\left\|f_{k}(z) ; H_{\mu(j)}\right\|^{2-2 \Lambda(r)}}\right)^{n-k}
$$

where $c_{k}>0$ is a constant. Since $\left\|f_{n} ; H_{\mu(j)}\right\|$ is a constant for any $0 \leq j \leq n$, we have

$$
\begin{aligned}
& \prod_{j=0}^{n} \frac{1}{\left\|f(z) ; H_{\mu(j)}\right\|^{2}}=\prod_{k=0}^{n-1} \prod_{j=0}^{n} \frac{\left\|f_{k+1}(z) ; H_{\mu(j)}\right\|^{2}}{\left\|f_{k}(z) ; H_{\mu(j)}\right\|^{2-2 \Lambda(r)}} \cdot \prod_{k=0}^{n-1} \prod_{j=0}^{n} \frac{1}{\left\|f_{k}(z) ; H_{\mu(j)}\right\|^{2 \Lambda(r)}} \\
\leq & c \prod_{k=0}^{n-1}\left(\sum_{j=0}^{n} \frac{\left\|f_{k+1}(z) ; H_{\mu(j)}\right\|^{2}}{\left\|f_{k}(z) ; H_{\mu(j)}\right\|^{2-2 \Lambda(r)}}\right)^{n-k} \cdot \prod_{k=0}^{n-1} \prod_{j=0}^{n} \frac{1}{\left\|f_{k}(z) ; H_{\mu(j)}\right\|^{2 \Lambda(r)}},
\end{aligned}
$$

where $c>1$ is a constant. Therefore, we have

$$
\begin{aligned}
& \int_{M\langle r\rangle} \max _{K} \sum_{j \in K} \log \frac{1}{\left\|f(z) ; H_{j}\right\|^{2}} \sigma \\
= & \int_{M\langle r\rangle} \max _{\mu \in T} \log \left(\prod_{j=0}^{n} \frac{1}{\left\|f(z) ; H_{\mu(j)}\right\|^{2}}\right) \sigma \\
\leq & \sum_{k=0}^{n-1} \int_{M\langle r\rangle} \max _{\mu \in T} \log \left(\sum_{j=0}^{n} \frac{\left\|f_{k+1}(z) ; H_{\mu(j)}\right\|^{2}}{\left\|f_{k}(z) ; H_{\mu(j)}\right\|^{2-2 \Lambda(r)}}\right)^{n-k} \sigma \\
& +\sum_{k=0}^{n-1} \sum_{j=0}^{n} \int_{M\langle r\rangle} \max _{\mu \in T} \log \frac{1}{\left\|f_{k}(z) ; H_{\mu(j)}\right\|^{2 \Lambda(r)}} \sigma+O(1) \\
= & \sum_{k=0}^{n-1}(n-k) \int_{M\langle r\rangle} \log \max _{\mu \in T}\left(\sum_{j=0}^{n} \frac{\left\|f_{k+1}(z) ; H_{\mu(j)}\right\|^{2}}{\left\|f_{k}(z) ; H_{\mu(j)}\right\|^{2-2 \Lambda(r)}} h_{k}^{2}\right) \sigma \\
& -2 \sum_{k=0}^{n-1}(n-k) S_{k}(r)+\sum_{k=0}^{n-1} \sum_{j=0}^{n} \int_{M\langle r\rangle} \max _{\mu \in T} \log \frac{1}{\left\|f_{k}(z) ; H_{\mu(j)}\right\|^{2 \Lambda(r)}} \sigma+O(1)
\end{aligned}
$$

where, in above, $h_{k}$ is defined by $(1.8), S_{k}(r)$ is defined by (1.9). We now estimate 
each term appearing the above inequality. First,

$$
\begin{aligned}
& \int_{M\langle r\rangle} \log \max _{\mu \in T}\left(\sum_{j=0}^{n} \frac{\left\|f_{k+1}(z) ; H_{\mu(j)}\right\|^{2}}{\left\|f_{k}(z) ; H_{\mu(j)}\right\|^{2-2 \Lambda(r)}} h_{k}^{2}\right) \sigma \\
= & \kappa \int_{M\langle r\rangle} \log \max _{\mu \in T}\left(\sum_{j=0}^{n} \frac{\left\|f_{k+1}(z) ; H_{\mu(j)}\right\|^{2}}{\left\|f_{k}(z) ; H_{\mu(j)}\right\|^{2-2 \Lambda(r)}} h_{k}^{2}\right) \frac{\sigma}{\kappa} \\
\leq & \kappa \log \int_{M\langle r\rangle} \max _{\mu \in T}\left(\sum_{j=0}^{n} \frac{\left\|f_{k+1}(z) ; H_{\mu(j)}\right\|^{2}}{\left\|f_{k}(z) ; H_{\mu(j)}\right\|^{2-2 \Lambda(r)}} h_{k}^{2}\right) \frac{\sigma}{\kappa} \\
\leq & \kappa \max _{1 \leq j \leq q} \log ^{+} \int_{M\langle r\rangle}\left(\frac{\left\|f_{k+1}(z) ; H_{j}\right\|^{2}}{\left\|f_{k}(z) ; H_{j}\right\|^{2-2 \Lambda(r)}} h_{k}^{2}\right) \sigma+C^{\prime} .
\end{aligned}
$$

By Theorem 3.7.3,

$$
\begin{aligned}
& \max _{1 \leq j \leq q} \log ^{+} \int_{M\langle r\rangle}\left(\frac{\left\|f_{k+1}(z) ; H_{j}\right\|^{2}}{\left\|f_{k}(z) ; H_{j}\right\|^{2-2 \Lambda(r)}} h_{k}^{2}\right) \sigma \\
\leq & 2 \log ^{+} T_{f}(r)+2 \log ^{+} \operatorname{Ric}_{\tau}(r)+\log ^{+} Y(r)+10 \log ^{+} \log T_{f}(r)+9 \log ^{+} \log ^{+} \operatorname{Ric}_{\tau}(r) \\
& +10 \log ^{+} \log ^{+} Y(r)+4 \log ^{+} \log r . \|_{E} .
\end{aligned}
$$

Hence,

$$
\begin{aligned}
& \sum_{k=0}^{n-1} \int_{M\langle r\rangle} \log \max _{\mu \in T}\left(\sum_{j=0}^{n} \frac{\left\|f_{k+1}(z) ; H_{\mu(j)}\right\|^{2}}{\left\|f_{k}(z) ; H_{\mu(j)}\right\|^{2-2 \Lambda(r)}} h_{k}^{2}\right)^{n-k} \sigma \\
\leq & n(n+1) \varsigma\left[\log ^{+} T_{f}(r)+\log ^{+} \operatorname{Ric}_{\tau}(r)+\frac{1}{2} \log ^{+} Y(r)+5 \log ^{+} \log T_{f}(r)\right. \\
& \left.+5 \log ^{+} \log ^{+} \operatorname{Ric}_{\tau}(r)+5 \log ^{+} \log ^{+} Y(r)+2 \log ^{+} \log r\right] \|_{E} .
\end{aligned}
$$

Next, using Theorem 3.5.1 (the Plücker formula), we have

$$
N_{l_{k}}(r)+T_{f_{k-1}}(r)-2 T_{f_{k}}(r)+T_{f_{k+1}}(r)=S_{k}(r)-S_{k}\left(r_{0}\right)+R i c_{\tau}(r) .
$$

Noticing that $T_{f_{n}}(r)=0$,

$$
\sum_{k=0}^{n-1}(n-k) S_{k}(r)=N_{d_{n}}(r)-(n+1) T_{f}(r)-\frac{n(n+1)}{2} R i c_{\tau}(r)+O(1)
$$


Finally, by the First Main Theorem,

$$
\begin{aligned}
& \sum_{k=0}^{n-1} \sum_{j=0}^{n} \int_{M\langle r\rangle} \max _{\mu \in T} \log \frac{1}{\left\|f_{k}(z) ; H_{\mu(j)}\right\|^{2 \Lambda(r)}} \sigma \\
\leq & \sum_{\mu \in T} \sum_{k=0}^{n-1} \sum_{j=0}^{n} \int_{M\langle r\rangle} 2 \Lambda(r) \log \frac{1}{\left\|f_{k}(z) ; H_{\mu(j)}\right\|} \sigma+O(1) \\
= & \sum_{\mu \in T} \sum_{k=0}^{n-1} \sum_{j=0}^{n} 2 \Lambda(r) m_{f_{k}}\left(r, H_{\mu(j)}\right)+O(1) \\
\leq & \sum_{k=0}^{n-1} \sum_{j=0}^{n} 2 q ! \Lambda(r)\left(T_{f_{k}}(r)+m_{f_{k}}\left(s_{0}, H_{\mu(j)}\right)\right)+O(1) \\
\leq & O(1) .
\end{aligned}
$$

Combining (1.12), (1.13), (1.14), and (1.15), we have

$$
\begin{aligned}
& \int_{M\langle r\rangle} \max _{K} \sum_{j \in K} \log \frac{1}{\left\|f(z) ; H_{j}\right\|} \sigma \leq(n+1) T_{f}(r)+\frac{n(n+1)}{2} \operatorname{Ric}_{\tau}\left(r, s_{0}\right)-N_{d_{n}}\left(r, s_{0}\right) \\
& +\varsigma \frac{n(n+1)}{2}\left[\log ^{+} T_{f}(r)+\log ^{+} \operatorname{Ric}_{\tau}(r)+\frac{1}{2} \log ^{+} Y(r)+5 \log ^{+} \log T_{f}(r)\right. \\
& \left.+5 \log ^{+} \log ^{+} \operatorname{Ric}_{\tau}(r)+5 \log ^{+} \log ^{+} Y(r)+3 \log ^{+} \log ^{+} r\right] \|_{E} .
\end{aligned}
$$

3.9. The main result. We are now ready to state the second result of our two main theorems in the paper which extends Theorem A to meromorphic mappings on parabolic manifolds.

THEOREM 2. Let $M$ be an admissible parabolic manifold of dimension $m$. Let $f: M \rightarrow X \subset \mathbb{P}^{N}(\mathbb{C})$ be an algebraically non-degenerate meromorphic map, where $X$ is a smooth algebraic variety. Let $D_{1}, \ldots, D_{q}$ be hypersurfaces in $\mathbb{P}^{N}(\mathbb{C})$ of degree $d_{j}$ in general position in $X$. Then for every $\epsilon>0$, we have

$$
\begin{aligned}
& \sum_{j=1}^{q} d_{j}^{-1} m_{f}\left(r, D_{j}\right) \leq(\operatorname{dim} X+1+\epsilon) T_{f}(r)+c_{1, \epsilon} \operatorname{Ric}_{\tau}(r) \\
& +c_{2, \epsilon}\left[\log ^{+} T_{f}(r)+\log ^{+} \operatorname{Ric}_{\tau}(r)+\frac{1}{2} \log ^{+} Y(r)+5 \log ^{+} \log ^{+} T_{f}(r)\right. \\
& \left.+5 \log ^{+} \log ^{+} \operatorname{Ric}_{\tau}(r)+5 \log ^{+} \log ^{+} Y(r)+3 \log ^{+} \log ^{+} r\right] \|_{E}
\end{aligned}
$$

where $E \subset\left[r_{0},+\infty\right)$ is a set with finite measure, $c_{1, \epsilon}$ and $c_{2, \epsilon}$ are two positive constants depending on $\epsilon$ which are explicitly given in the proof below.

We note that $\operatorname{Ric}_{\tau}(r)$ is called the Ricci function which depends only on the geometry (topology) of the manifold $M$. An important class of admissible parabolic manifolds consists of affine algebraic manifolds. In this case, there exists a finite branched covering $\pi: M \rightarrow \mathbb{C}^{m}$. If we take $\tau=\|\pi\|^{2}$, Stoll [23] showed that there exists a holomorphic differential form $B$ of degree $(m-1,0)$ on $M$ (c.f. [23] or [27]) such that

$$
m i_{m-1} B \wedge \bar{B} \leq(1+|\tau|)^{m-1}\left(d d^{c} \tau\right)^{m-1}
$$


Hence, we can take $Y(r)=\left(1+r^{2}\right)^{m-1}$. Further, we can show (c.f. [23] or [27]) that

$$
d_{\pi}=\lim _{r \rightarrow+\infty} \frac{R i c_{\tau}\left(r, s_{0}\right)}{\log r}
$$

exists and equals the degree of the branching divisor of $\pi$. The Theorem 2 thus implies the following corollary in the case that $M$ is affine algebraic manifold.

COROllary. Let $M$ be an affine algebraic manifold of complex dimension $m$. Let $\pi: M \rightarrow \mathbb{C}^{m}$ be a finite branched covering. Let $f: M \rightarrow X \subset \mathbb{P}^{N}(\mathbb{C})$ be an algebraically non-degenerate meromorphic map. Let $D_{1}, \ldots, D_{q}$ be a finite collection of hypersurfaces in $\mathbb{P}^{N}(\mathbb{C})$ in general position in $X$. Assume that $f$ is transcendental. Then

$$
\sum_{j=1}^{q} \delta_{f}\left(D_{j}\right) \leq \operatorname{dim} X+1
$$

Proof of Theorem 2. For given hypersurfaces $D_{1}, \ldots, D_{q}$ defined by $Q_{1}, \ldots, Q_{q}$, assuming they have the same degree (WLOG), say $d$. The proof depends on the Key inequality (see below) which allows us to the transfer from the (study of ) hypersurfaces to the hyperplanes. To state and prove the Key inequality, we consider the map $\chi: X \rightarrow \mathbb{P}^{q-1}(\mathbb{C})$ defined by $\chi(w)=\left[Q_{1}(w): \cdots: Q_{q}(w)\right]$. It is a finite morphism since $D_{1}, \ldots D_{q}$ are in general position. Let $Z=\chi(X)$. We have that $Z$ is a complex subvariety of $\mathbb{P}^{q-1}, \operatorname{dim} Z=\operatorname{dim} X=n$ and

$$
\triangle:=\operatorname{deg} Z \leq d^{n} \operatorname{deg} X .
$$

We shall study the map $\chi \circ f: \mathbb{C} \rightarrow Z \subset \mathbb{P}^{q-1}(\mathbb{C})$.

We first introduce some notation: Let $Z \subset \mathbb{P}^{l}$ be a projective variety of dimension $n$ (with $l=q-1$ in the above case). Denote by $\mathbb{C}\left[x_{0}, \ldots, x_{l}\right]_{m}$ the vector space of homogeneous polynomials in $\mathbb{C}\left[x_{0}, \ldots, x_{l}\right]$ of degree $\mathrm{m}$ (including 0 ). Let $I_{Z}$ be the prime ideal in $\mathbb{C}\left[x_{0}, \ldots, x_{l}\right]$ defining $Z$. Put $\left(I_{Z}\right)_{m}=\mathbb{C}\left[x_{0}, \ldots, x_{l}\right]_{m} \cap I_{Z}$. The Hilbert polynomial $H_{Z}(m)$ of $Z$ is defined by

$$
H_{Z}(m):=\operatorname{dim}\left(\mathbb{C}\left[x_{0}, \ldots, x_{l}\right]_{m} / I_{Z}(m)\right) .
$$

The Hilbert Weight of $Z$ with respect to the weight $\mathbf{c} \in \mathbb{R}^{l+1}$ is defined by

$$
S_{Z}(m, \mathbf{c})=\max \left(\sum_{i=1}^{H_{Z}(m)} \mathbf{a}_{i} \cdot \mathbf{c}\right)
$$

where the maximum is taken over all sets of monomials $\mathbf{x}^{\mathbf{a}_{1}}, \ldots, \mathbf{x}^{\mathbf{a}_{H_{Z}(m)}}$ whose residue classes modulo $I_{Z}$ form a basis of $\mathbb{C}\left[x_{0}, \ldots, x_{l}\right]_{m} /\left(I_{Z}\right)_{m}$, where for $\mathbf{x}=\left(x_{0}, \ldots, x_{l}\right)$, $\mathbf{a}=\left(a_{0}, \ldots, a_{l}\right) \in \mathbb{Z}_{+}^{l+1}, \mathbf{x}^{\mathbf{a}}=x_{0}^{a_{0}} \cdots x_{l}^{a_{l}}$. We need the following lemma to estimate (get a lower bound) of Hilbert Weight $S_{Z}(m, \mathbf{c})$.

Lemma 3.9.1 (see [18]). Let $Z \subset \mathbb{P}^{l}$ be an algebraic variety of dimension $n$ and degree $\triangle$. Let $m>\triangle$ be an integer and let $\mathbf{c}=\left(c_{0}, \ldots, c_{l}\right) \in \mathbb{R}^{l+1}$. Let $\left\{i_{0}, \ldots, i_{n}\right\}$ be a subset of $\{0, \ldots, l\}$ such that

$$
\left\{x=\left[x_{0}: \cdots: x_{l}\right) \in \mathbb{P}^{l} \mid x_{i_{0}}=\cdots=x_{i_{n}}=0\right\} \cap Z=\emptyset .
$$


Then

$$
\frac{1}{m H_{Z}(m)} S_{Z}(m, \mathbf{c}) \geq \frac{1}{(n+1)}\left(c_{i_{0}}+\cdots+c_{i_{n}}\right)-\frac{(2 n+1) \triangle}{m}\left(\max _{0 \leq i \leq l} c_{i}\right) .
$$

Now with the above $Z=\chi(X) \subset \mathbb{P}^{q-1}$, consider the vector space $V_{m}=$ $\mathbb{C}\left[x_{1}, \ldots, x_{q}\right]_{m} /\left(I_{Z}\right)_{m}$, and fix a basis $\phi_{0}, \ldots, \phi_{n_{m}}$ where $n_{m}=H_{Z}(m)-1$. Consider the map

$$
F=\left[\phi_{0}(\chi \circ f): \cdots: \phi_{n_{m}}(\chi \circ f)\right]: M \rightarrow \mathbb{P}^{n_{m}} .
$$

Let $\left\{U_{\lambda}, \lambda \in \Lambda\right\}$ be an open covering of $M$, and let $\mathbf{f}_{\lambda}: U_{\lambda} \rightarrow \mathbb{C}^{N+1}$ be a reduced representation of $f$ on $U_{\lambda}$. Write, on $U_{\lambda}, \mathbf{F}_{\lambda}=\left(F_{0, \lambda}, \ldots, F_{n_{m}, \lambda}\right)$ where $F_{i, \lambda}=\phi_{i}\left(Q_{1}(\mathbf{f}), \ldots, Q_{q}(\mathbf{f})\right)$ for $i=0,1, \ldots, n_{m}$. Note that $\mathbf{F}_{\lambda}$ is a reduced representation of $F$ on $U_{\lambda}$. Define,

$$
c_{j}(z)=\log \frac{\left\|\mathbf{f}_{\lambda}(z)\right\|^{d}\left\|Q_{j}\right\|}{\left|Q_{j}\left(\mathbf{f}_{\lambda}\right)(z)\right|}, 1 \leq j \leq q,
$$

which, we note that, is independent of the choice of $f_{\lambda}$ (hence is independent of $\lambda)$, so $c_{j}$ are globally defined functions on $M$. Let $\mathbf{c}(z)=\left(c_{1}(z), \ldots, c_{q}(z)\right)$. With this given weight $\mathbf{c}(z)$, take monomials (which depends on $z$ ) $\mathbf{x}^{\mathbf{a}_{1}}, \ldots, \mathbf{x}^{\mathbf{a}_{H_{Z}(m)}}$ whose residue classes modulo $I_{Z}$ form a basis of $V_{m}=\mathbb{C}\left[x_{1}, \ldots, x_{q}\right]_{m} /\left(I_{Z}\right)_{m}$ and which reaches the max. in the Hilbert weight $S_{Z}(m, \mathbf{c}(z))$. For each $1 \leq j \leq H_{Z}(m)$, write $\mathbf{x}^{\mathbf{a}_{j}}=L_{j, z}\left(\phi_{0}, \ldots \phi_{n_{m}}\right)$ then, for $L_{j, z}$ are linear forms which are linearly independent for each fixed $z$. Note that the linear forms depends on $z$, but there are only finitely many of them in total. We then have the following key inequality which transfers the study of hyerpsurfaces for $f$ into the study of linear forms for the map $F$ (so we can apply Cartan's result for $F$ ).

Key Inequality. For each $z \in U_{\lambda}$ and linear forms $L_{j, z}, 1 \leq j \leq H_{Z}(m)=$ $n_{m}+1$, chosen above, we have

$$
\begin{aligned}
\sum_{j=1}^{q} \log \frac{\left\|\mathbf{f}_{\lambda}(z)\right\|^{d}\left\|Q_{j}\right\|}{\left|Q_{j}\left(\mathbf{f}_{\lambda}\right)(z)\right|} & \leq \frac{n+1}{m H_{Z}(m)} \sum_{j=1}^{H_{Z}(m)} \log \frac{\left\|\mathbf{F}_{\lambda}(z)\right\|}{\left|L_{j, z}\left(\mathbf{F}_{\lambda}\right)(z)\right|} \\
& +\frac{(2 n+1) \triangle}{m}\left(\max _{0 \leq i \leq q} \log \frac{\left\|\mathbf{f}_{\lambda}(z)\right\|\left\|Q_{i}\right\|}{\left|Q_{i}\left(\mathbf{f}_{\lambda}\right)(z)\right|}\right)+O(1)
\end{aligned}
$$

where $O(1)$ denotes a bounded term which is independent of $z$.

We note that terms on both left and right hand sides are is independent of the choice of $\mathbf{f}_{\lambda}$ (hence is independent of $\lambda$ ). Thus the inequality indeed holds on $M$.

Proof. For every $\mathbf{b}=\left[b_{0}: \cdots: b_{N}\right] \in \mathbb{P}^{N}(\mathbb{C})$, consider the function

$$
\left\|\mathbf{b}, D_{j}\right\|=\frac{\left|Q_{j}(\mathbf{b})\right|}{\|\mathbf{b}\|^{d}\left\|Q_{j}\right\|}
$$

where $\|\mathbf{b}\|=\max _{0 \leq j \leq N}\left|b_{j}\right|$ and $\left\|Q_{j}\right\|$ is the maximum of the absolute values of the coefficients of $Q_{j}$. By the "in general position" condition, at each point $\mathbf{b} \in X$, there $\left\|\mathbf{b}, D_{j}\right\|$ can be zero for no more than $n$ indicies $j \in\{1, \ldots, q\}$. For the remaining indices $j$, we have $\left\|\mathbf{b}, D_{j}\right\|>0$ and by the continuity of these functions and the 
compactness of $V$, there exists $C>0$ such that $\left\|\mathbf{b}, D_{j}\right\|>C$ for all $\mathbf{b} \in V$ and all $D_{j}$, except for at most $n$ of them. Hence, for $z \in U_{\lambda}$, there is $i_{0}, \ldots, i_{n}$ (which may depend on $z$ ) such that

$$
\sum_{j=1}^{q} \log \frac{\left\|\mathbf{f}_{\lambda}(z)\right\|^{d}\left\|Q_{j}\right\|}{\left|Q_{j}\left(\mathbf{f}_{\lambda}\right)(z)\right|} \leq \sum_{t=0}^{n} \log \frac{\left\|\mathbf{f}_{\lambda}(z)\right\|^{d}\left\|Q_{i_{t}}\right\|}{\left|Q_{i_{t}}\left(\mathbf{f}_{\lambda}\right)(z)\right|}
$$

With $c_{j}(z)=\log \frac{\left\|\mathbf{f}_{\lambda}(z)\right\|^{d}\left\|Q_{j}\right\|}{\left|Q_{j}\left(\mathbf{f}_{\lambda}\right)(z)\right|}$ and $\mathbf{c}(z)=\left(c_{1}(z), \ldots, c_{q}(z)\right)$, and by applying Lemma 3.9.1, we get

$$
\frac{1}{m H_{Z}(m)} S_{Z}(m, \mathbf{c}(z)) \geq \frac{1}{(n+1)}\left(c_{i_{0}}(z)+\cdots+c_{i_{n}}(z)\right)-\frac{(2 n+1) \triangle}{m}\left(\max _{1 \leq i \leq q} c_{i}(z)\right)
$$

which gives

$\sum_{t=0}^{n} \log \frac{\left\|\mathbf{f}_{\lambda}(z)\right\|^{d}\left\|Q_{i_{t}}\right\|}{\left|Q_{i_{t}}\left(\mathbf{f}_{\lambda}\right)(z)\right|} \leq \frac{(n+1) S_{Z}(m, \mathbf{c}(z))}{m H_{Z}(m)}+\frac{(n+1)(2 n+1) \triangle}{m}\left(\max _{0 \leq i \leq q} \log \frac{\left\|\mathbf{f}_{\lambda}(z)\right\|\left\|Q_{i}\right\|}{\left|Q_{i}\left(\mathbf{f}_{\lambda}\right)(z)\right|}\right)$.

Therefore,

$\sum_{j=1}^{q} \log \frac{\left\|\mathbf{f}_{\lambda}(z)\right\|^{d}\left\|Q_{j}\right\|}{\left|Q_{j}\left(\mathbf{f}_{\lambda}\right)(z)\right|} \leq \frac{(n+1) S_{Z}(m, \mathbf{c}(z))}{m H_{Z}(m)}+\frac{(n+1)(2 n+1) \triangle}{m}\left(\max _{0 \leq i \leq q} \log \frac{\left\|\mathbf{f}_{\lambda}(z)\right\|^{d}\left\|Q_{i}\right\|}{\left|Q_{i}\left(\mathbf{f}_{\lambda}\right)(z)\right|}\right)$.

But by our selection of the linear forms $L_{j, z}, L_{j, z}\left(\phi_{0}, \ldots \phi_{n_{m}}\right)=\mathbf{x}^{\mathbf{a}_{j}}$ and

$$
S_{Z}(m, \mathbf{c}(z))=\sum_{i=1}^{H_{Z}(m)} \mathbf{a}_{i} \cdot \mathbf{c}(z)
$$

Notice that from $L_{i, z}\left(\phi_{0}, \ldots \phi_{n_{m}}\right)=\mathbf{x}^{\mathbf{a}_{i}}$, we get $L_{i, z}\left(\mathbf{F}_{\lambda}(z)\right)=$ $\left.\left(Q_{1}\left(\mathbf{f}_{\lambda}\right)(z)\right)^{a_{i 1}} \cdots Q_{q}\left(\mathbf{f}_{\lambda}\right)(z)\right)^{a_{i q}}$ where $\mathbf{a}_{i}=\left(a_{i 1}, \ldots, a_{i q}\right)$. So

$$
\begin{aligned}
-\log \left|L_{i, z}\left(\mathbf{F}_{\lambda}(z)\right)\right| & =-\left(a_{i 1} \log \left|Q_{1}\left(\mathbf{f}_{\lambda}\right)(z)\right|+\cdots+a_{i 1} \log \left|Q_{q}\left(\mathbf{f}_{\lambda}\right)(z)\right|\right) \\
& =a_{i 1} c_{1}(z)+\cdots+a_{i q} c_{q}(z)-m \log \left\|\mathbf{f}_{\lambda}(z)\right\|^{d} \\
& =\mathbf{a}_{i} \cdot \mathbf{c}(z)-m \log \left\|\mathbf{f}_{\lambda}(z)\right\|^{d} .
\end{aligned}
$$

Thus

$$
-\sum_{i=1}^{H_{Z}(m)} \log \left|L_{i, z}\left(\mathbf{F}_{\lambda}(z)\right)\right|=S_{Z}(m, \mathbf{c}(z))-m H_{Z}(m) \log \left\|\mathbf{f}_{\lambda}(z)\right\|^{d}
$$

Hence

$$
\begin{aligned}
& \sum_{j=1}^{q} \log \frac{\left\|\mathbf{f}_{\lambda}(z)\right\|^{d}\left\|Q_{j}\right\|}{\left|Q_{j}\left(\mathbf{f}_{\lambda}\right)(z)\right|} \leq \frac{(n+1)}{m H_{Z}(m)} \sum_{i=1}^{H_{Z}(m)} \log \frac{\left\|\mathbf{F}_{\lambda}(z)\right\|}{\left|L_{i, z}\left(\mathbf{F}_{\lambda}\right)(z)\right|}+\frac{n+1}{m} \log \frac{\left\|\mathbf{f}_{\lambda}(z)\right\|^{d m}}{\left\|\mathbf{F}_{\lambda}(z)\right\|} \\
& +\frac{(n+1)(2 n+1) \triangle}{m}\left(\max _{0 \leq i \leq q} \log \frac{\left\|\mathbf{f}_{\lambda}(z)\right\|^{d}\left\|Q_{i}\right\|}{\left|Q_{i}\left(\mathbf{f}_{\lambda}\right)(z)\right|}\right) .
\end{aligned}
$$

By the definition of $\mathbf{F}$, we easily see that $C_{1}\left\|\mathbf{f}_{\lambda}\right\|^{d m} \leq\left\|\mathbf{F}_{\lambda}\right\| \leq C_{2}\left\|\mathbf{f}_{\lambda}\right\|^{d m}$ for some positive $C_{1}$ and $C_{2}$ independent of $\lambda$. This proves our key inequality. 
We now continue our proof. By integrating the key inequality and applying Theorem 3.8.1 to $F$, we get

$$
\begin{aligned}
& \sum_{j=1}^{q} m_{f}\left(r, D_{j}\right) \leq \frac{n+1}{m H_{Y}(m)}\left\{\left(n_{m}+1\right) T_{F}(r)+\frac{n_{m}\left(n_{m}+1\right)}{2} \operatorname{Ric}_{\tau}(r)\right. \\
& +\varsigma \frac{n_{m}\left(n_{m}+1\right)}{2}\left[\log ^{+} T_{F}(r)+\log ^{+} \operatorname{Ric}_{\tau}(r)+\frac{1}{2} \log ^{+} Y(r)+5 \log ^{+} \log T_{F}(r)\right. \\
& \left.\left.+5 \log ^{+} \log ^{+} \operatorname{Ric}_{\tau}(r)+5 \log ^{+} \log ^{+} Y(r)+3 \log ^{+} \log ^{+} r\right]\right\} \|_{E} \\
& +\frac{(2 n+1)(n+1) \triangle}{m} \int_{0}^{2 \pi}\left(\max _{1 \leq j \leq q} \log \frac{\left\|\mathbf{f}_{\lambda}^{d}\left(r e^{i \theta}\right)\right\|\left\|Q_{j}\right\|}{\mid Q_{j}\left(\mathbf{f}_{\lambda}\left(r e^{i \theta}\right) \mid\right.}\right) \frac{d \theta}{2 \pi}+O(1) .
\end{aligned}
$$

For each $j \in\{1, \ldots, q\}$, by the First Main Theorem,

$$
\int_{0}^{2 \pi} \log \frac{\left\|f\left(r e^{i \theta}\right)\right\|\left\|Q_{j}\right\|}{\left|Q_{j}\left(r e^{i \theta}\right)\right|} \frac{d \theta}{2 \pi} \leq d T_{f}(r)+O(1) .
$$

It remains to compare $T_{f}(r)$ and $T_{F}(r)$. Let $\left\{U_{\lambda}, \lambda \in \Lambda\right\}$ be an open covering of $M$, and let $\mathbf{f}_{\lambda}: U_{\lambda} \rightarrow \mathbb{C}^{N+1}$ be a reduced representation of $f$ on $U_{\lambda}$, then there is a holomorphic function $g_{\lambda \mu}: U_{\lambda} \cap U_{\mu} \rightarrow \mathbb{C}_{*}$ such that

$$
\mathbf{f}_{\lambda}=g_{\lambda \mu} \mathbf{f}_{\mu} \quad \text { on } \quad U_{\lambda} \cap U_{\mu} .
$$

It is easy to check that $\left\{g_{\lambda \mu}\right\}$ is a basic cocycle (cf. [23]). Therefore there exists a holomorphic line bundle $L_{f}$ on $M$ with a holomorphic frame atlas $\left\{U_{\lambda}, s_{\lambda}\right\}_{\lambda \in \Lambda}$ such that

$$
s_{\lambda}=g_{\mu \lambda} v_{\mu} \quad \text { on } \quad U_{\lambda} \cap U_{\mu} .
$$

The line bundle $L_{f}$ is called the hyperplane section bundle of $f$. Also define $\tilde{\mathbf{f}}_{\lambda} \in$ $\Gamma\left(U_{\lambda}, M \times \mathbb{C}^{N+1}\right)$ by $\tilde{\mathbf{f}}_{\lambda}(z)=\left(z, \mathbf{f}_{\lambda}(z)\right)$ for $z \in U_{\lambda}$. Notice that $\tilde{\mathbf{f}}_{\lambda} \otimes s_{\lambda}=g_{\lambda \mu} \tilde{\mathbf{f}}_{\mu} \otimes s_{\lambda}=$ $\tilde{\mathbf{f}}_{\mu} \otimes g_{\lambda \mu} s_{\lambda}=\tilde{\mathbf{f}}_{\mu} \otimes s_{\mu}$ on $U_{\lambda} \cap U_{\mu}$. Therefore there exists a holomorphic section $\Gamma_{f}$ of $\left(M \times \mathbb{C}^{N+1}\right) \otimes L_{f}$ such that $\left.\Gamma_{f}\right|_{U_{\lambda}}=\tilde{\mathbf{f}}_{\lambda} \otimes s_{\lambda} . \Gamma_{f}$ is called the standard reduced representation section of $f$. Let $\ell$ be the standard hermitian metric along the fibers of the trivial bundle $M \times \mathbb{C}^{N+1}$ and $\kappa$ be a hermitian metric along the fibers of $L_{f}$. Then

$$
d d^{c} \log \left\|\Gamma_{f}\right\|_{\ell \otimes \kappa}^{2}=d d^{c} \log \left\|\mathbf{f}_{\lambda}\right\|^{2}+d d^{c} \log \left\|s_{\lambda}\right\|_{\kappa}^{2}=f^{*} \Omega_{F S}-c_{1}\left(L_{f}, \kappa\right),
$$

where $\Omega_{F S}$ is the Fubini-Study form on $\mathbb{P}^{N}(\mathbb{C})$. Hence, by Green's formula(cf. [23]), we have

$$
\begin{aligned}
T_{f}(r)= & \int_{r_{0}}^{r} \frac{d t}{t^{2 m-1}} \int_{M[t]} f^{*} \Omega_{F S} \wedge v^{m-1}=\int_{r_{0}}^{r} \frac{d t}{t^{2 m-1}} \int_{M[t]} f^{*} c_{1}\left(L_{f}, \kappa\right) \wedge v^{m-1} \\
& +\int_{M\langle r\rangle} \log \left\|\Gamma_{f}\right\|_{\ell \otimes \kappa}-\int_{M<r_{0}>} \log \left\|\Gamma_{f}\right\|_{\ell \otimes \kappa} \sigma .
\end{aligned}
$$

Similarly,

$$
\begin{aligned}
T_{F}(r)= & \int_{r_{0}}^{r} \frac{d t}{t^{2 m-1}} \int_{M[t]} f^{*} c_{1}\left(L_{F}, \kappa\right) \wedge v^{m-1} \\
& +\int_{M\langle r\rangle} \log \left\|\Gamma_{F}\right\|_{\ell \otimes \kappa}-\int_{M<r_{0}>} \log \left\|\Gamma_{F}\right\|_{\ell \otimes \kappa} \sigma .
\end{aligned}
$$


By comparing the transition functions of $L_{f}$ and $L_{F}$, it is clear that $L_{F}=L_{f}^{\otimes d m}$ and also

$$
\frac{\left\|\Gamma_{F}\right\|_{\ell \otimes \kappa}}{\left\|\Gamma_{L}\right\|_{\ell \otimes \kappa}^{d m}}=\frac{\left\|\mathbf{F}_{\lambda}\right\|}{\left\|\mathbf{f}_{\lambda}\right\|^{d m}} \leq C .
$$

We thus conclude that

$$
T_{F}(r) \leq d m T_{f}(r)+O(1)
$$

By combing (1.16), (1.17) and (1.18), we get, noticing that $H_{Z}(m)=n_{m}+1$,

$$
\begin{aligned}
& \sum_{j=1}^{q} m_{f}\left(r, D_{j}\right) \leq(n+1) d T_{f}(r)+\frac{(n+1) n_{m}}{2 m} \operatorname{Ric}_{\tau}(r) \\
& +\frac{\varsigma n_{m}}{2 m}\left[\log ^{+} T_{f}(r)+\log ^{+} \operatorname{Ric}_{\tau}(r)+\frac{1}{2} \log ^{+} Y(r)+5 \log ^{+} \log ^{+} T_{f}(r)\right. \\
& \left.+5 \log ^{+} \log ^{+} \operatorname{Ric}_{\tau}(r)+5 \log ^{+} \log ^{+} Y(r)+3 \log ^{+} \log ^{+} r\right] \\
& +\frac{(2 n+1)(n+1) d \triangle}{m} T_{f}(r) \|_{E} .
\end{aligned}
$$

For any given $\epsilon>0$, take $m_{0}$ such that $\frac{(2 n+1)(n+1) \triangle}{m_{0}}<\epsilon$, then

$$
\begin{aligned}
& \sum_{j=1}^{q} m_{f}\left(r, D_{j}\right) \leq(n+1+\epsilon) d T_{f}(r)+\frac{(n+1) n_{m_{0}}}{2 m_{0}} \operatorname{Ric}_{\tau}(r) \\
& +\frac{\varsigma n_{m_{0}}}{2 m_{0}}\left[\log ^{+} T_{f}(r)+\log ^{+} \operatorname{Ric}_{\tau}(r)+\frac{1}{2} \log ^{+} Y(r)+5 \log ^{+} \log ^{+} T_{f}(r)\right. \\
& \left.+5 \log ^{+} \log ^{+} \operatorname{Ric}_{\tau}(r)+5 \log ^{+} \log ^{+} Y(r)+3 \log ^{+} \log ^{+} r\right] \|_{E} .
\end{aligned}
$$

This proves Theorem 2 with

$$
c_{1, \epsilon}=\frac{(n+1) n_{m_{0}}}{2 m_{0}}, c_{2, \epsilon}=\frac{\varsigma n_{m_{0}}}{2 m_{0}} .
$$

\section{REFERENCES}

[1] L. Ahlfors, The theory of meromorphic curves, Acta. Soc. Sci. Fenn. Nova Ser. A, 3 (1941), pp. $171-183$.

[2] W. BARTH, Transplanting cohomology classes in complex-projective space, Amer. J. Math., 92 (1970), pp. 951-967.

[3] J. Carlson And P. GRiffiths, A defect relation for equidimensional holomorphic mappings between algebraic vaieties, Ann. of Math., 95 (1972), pp. 557-584.

[4] .H. CARTAN, Sur les zeros des combinaisions linearires de p fonctions holomorpes donnees, Mathematica(Cluj), 7 (1933), pp. 80-103.

[5] P. Corvaja and U. M. Zannier, On a general Thue's equation, Amer. J. Math., 126 (2004), pp. 1033-1055.

[6] J.-H. Evertse And R. G. Ferretti, Diophantine inequalities on projective varieties, Int. Math. Res. Notices, 25 (2002), pp. 1295-1330.

[7] J.-H. Evertse And R. G. Ferretti, A generalization of the subspace theorem with polynomials of higher degree, In: Diophantine Approximation, Festschrift for Wolfgang Schmidt, R. F. Tichy, H. P. Schlikewei, K. Schmidt (eds), Proceedings of a conference in honor of Prof. Wolfgang Schmidt's 70th birthday, held in Vienna, October 6-10, 2003, Springer Verlag, 2008, pp. 175-198. 
[8] G. HeIER AND M. Ru, Essentially large divisors and their arithmetic and function-theoretic inequalities, Asian J. Math., 16 (2012), pp. 387-408.

[9] P. Griffiths And J. King, Nevanlinna theory and holomorphic mappings between algebraic varieties, Acta Math., 130 (1973), pp. 145-220.

[10] R. Hartshorne, Varieties of small codimension in projective space, Bull. Amer. Math. Soc., 80 (1974), pp. 1017-1032.

[11] R. Hartshorne, Algebraic Geometry, Grad. Texts in Math., vol. 52, Springer-Verlag, New York, 1997.

[12] M. LARSEn, On the topology of complex projective manifolds, Invent. Math., 19 (1973), pp. 251260.

[13] R. LAzArsfeld, Positivity in algebraic geometry. I, volume 48 of Ergebnisse der Mathematik und ihrer Grenzgebiete. 3. Folge. A Series of Modern Surveys in Mathematics, SpringerVerlag, Berlin, 2004.

[14] A. OGus, Local cohomological dimension of algebraic varieties, Ann. of Math. (2), 98 (1973), pp. 327-365.

[15] M. RU, The second main theorem with moving targets on parabolic manifolds, Indiana University Math. J., 46 (1997), pp. 299-318.

[16] M. Ru, On a general form of the second main theorem, Trans. Amer. Math. Soc., 349 (1997), pp. 5093-5105.

[17] M. Ru, A defect relation for holomorphic curves intersecting hypersurfaces, Amer. J. Math., 126 (2004), pp. 215-226.

[18] M. Ru, Holomorphic curves into algebraic varieties, Annals of Mathematics, 169 (2009), pp. 255-267.

[19] M. RU AND Y. LiU, A defect relation for meromorphic maps on parabolic manifolds intersecting hypersurfaces, Illinois J. Math., 49 (2005), pp. 237-257.

[20] M. Ru And J. Wang, A Second Main Theorem on parabolic manifolds, Asian J. Math., 9 (2005), pp. 349-371.

[21] W. Stoll, Die beiden Hauptsätze der Weitverteilungs-theorie dei Funktionen mehrerer komplexer Veränderlichen, I, Acta Math., 90 (1950), pp. 1-115; II ibid, 92 (1954), pp. 55-169.

[22] W. Stoll, Mehrfahe Integrale auf komplexan Mannig-faltigkeiten, Math. Z., 57 (1952), pp. $116-154$.

[23] W. Stoll, The Ahlfors-Weyl theory of meromorphic maps on parabolic manifolds, SpringerVerlag Lecture Notes in Mathematics, 981 (1983), pp. 101-219.

[24] P. VojtA, Cartan's theorem and Cartan's conjecture, Amer. J. Math., 119 (1997), pp. 1-17.

[25] H. Weyl And J. Weyl, Meromorphic functions and analytic curves, Princeton Univ. Press, (1943).

[26] P. M. Wong, Defect relations for maps on parabolic spaces and Kobayashi metrics on projective spaces omitting hyperplanes, Notre Dame Thesis (1976).

[27] P. M. Wong And W. Stoll, Second main theorem of Nevanlinna theory for nonequidimensional meromorphic maps, American J. Math., 116 (1994), pp. 1031-1071.

[28] H. Wu, The equidistribution theory of holomorphic curves, Ann. of Math. Studies, 64, Princeton University Press (1970). 